\title{
Environmental effects on the globular cluster blue straggler population: a statistical approach ${ }^{\star}$
}

\author{
A. Moretti ${ }^{1,3}$, F. De Angeli ${ }^{2}$, and G. Piotto ${ }^{1}$ \\ 1 Dipartimento di Astronomia - Università di Padova, Vicolo dell’Osservatorio 3, 35122 Padova, Italy \\ e-mail: alessia.moretti@unipd.it \\ 2 Institute of Astronomy, Madingley Road, CB3 0HA Cambridge, UK \\ 3 INAF - Osservatorio Astronomico di Padova, Vicolo dell'Osservatorio 5, 35122 Padova, Italy
}

Received 3 August 2007 / Accepted 3 March 2008

\section{ABSTRACT}

Context. Blue stragglers stars (BSS) constitute an ubiquitous population of objects whose origin involves both dynamical and stellar evolution.

Aims. In this paper we study the properties of a catalogue of BSS extracted from an homogeneous sample of 56 Galactic globular clusters (GC) observed with Wide-Field Planetary Camera 2 on board of Hubble Space Telescope (WFPC2/HST).

Methods. With the purpose of investigating the environmental dependence of the BSS formation mechanisms, we explore possible monovariate relations between the frequency of BSS (divided in different subsamples according to their location with respect to the parent cluster core radius and half-mass radius) and the main parameters of their host GC. We also performed a principal component analysis (PCA) to extract the main parent cluster parameters, which characterise the BSS family.

Results. We find that any subpopulation of BSS strongly depends on the luminosity of the cluster, on the extension of the cluster horizontal branch, and on the central velocity dispersion: more luminous clusters and clusters with a smaller central velocity dispersion have a higher BSS frequency. Moreover, we find that clusters having higher mass, higher central densities, and smaller core relaxation timescales have, on average, more luminous BSS. Finally, different dependencies seem to hold for clusters with different integrated luminosity: brighter clusters show a BSS population that depends on the collisional parameter, while BSS in fainter clusters are mostly influenced by the cluster luminosity and the dynamical timescales.

Key words. stars: blue stragglers - stars: luminosity function, mass function - stars: Hertzsprung-Russell (HR) and C-M diagrams Galaxy: globular clusters: general

\section{Introduction}

Starting from their early discovery by Sandage (1953), blue straggler stars (BSS) have been detected in all stellar associations, from open (Ahumada \& Lapasset 1995, 2007; de Marchi et al. 2006) to globular clusters (GC, see Piotto et al. 2004, for a recent compilation of an homogeneous sample of BSS in GC), to dwarf galaxies (Momany et al. 2007).

BSS are located blue-ward and at brighter magnitudes than the turnoff (TO) in the color-magnitude diagram (CMD). The simple presence of such stars in a CMD poses serious challenges to the stellar evolution theory, since cluster stars with masses higher than the TO mass should have already been evolved off the main sequence (MS) toward the red giant branch (RGB).

Explanations of the existence of these objects must take into account both the dynamical processes happening during the cluster lifetime and the stellar evolution itself. Two different mechanisms have been proposed so far to account for BSS existence: one describes BSS as the by-product of primordial binaries that simply evolve transferring their masses up to a complete coalescence (McCrea 1964; Carney et al. 2001), while the other one

\footnotetext{
* The tables with the BSS catalogues, similar to Table A.1, are only available in electronic form at the CDS via anonymous ftp to cdsarc.u-strasbg.fr $(130.79 .128 .5)$ or via
}

http://cdsweb.u-strasbg.fr/cgi-bin/qcat?J/A+A/483/183 predicts that BSS are formed from the merger (collision) of two main sequence stars during the dynamical evolution of the cluster (Bailyn 1995). We will refer to BSS of the first and second types as primordial and collisional BSS. Recent results show that both formation mechanisms could be at work in a given cluster (Ferraro et al. 1997; Piotto et al. 2004; Davies et al. 2004; Mapelli et al. 2004, 2006).

On the basis of the high resolution Hubble Space Telescope (HST) observations of GC cores, Piotto et al. (2004) found that the relative number of BSS in the central regions shows an anticorrelation with the absolute luminosity (and hence mass) of the host cluster, while the trend with the collision rate is not significant and there is no clear dependency on the central density. Post core-collapse (PCC) clusters present a central BSS population that is not different from the one shown by normal clusters. A similar trend between the BSS frequency and the total cluster luminosity has also been confirmed in open clusters (de Marchi et al. 2006).

A distinction in the BSS luminosity function was found when dividing clusters according to their integrated luminosity: more luminous GC have brighter BSS. The distinction is more evident when the cut in luminosity is set to $M_{V}=-8.8$. This value is in agreement with the estimated luminosity beyond which BSS are predominantly collisional, according to the model presented by Davies et al. (2004). 
Finally, Leigh et al. (2007) using the Padova GC database, tried to correlate the BSS frequency with the other cluster parameters, and found that, besides the luminosity, only the central velocity dispersion and the half-mass relaxation time have some influence on the BSS population. The lack of any correlation with the collisional parameters led the authors to claim that BSS should be mostly born from binary systems and not from star encounters.

Wide-field studies show a clear bimodality in the radial distribution of BSS, probably due to different formation mechanisms playing a prominent role in different environments. The first evidence of the presence of a BSS bimodal distribution (Ferraro et al. 1997) was found in M 3 and was later on confirmed for other clusters: M 55 (Zaggia et al. 1997); 47 Tuc (Ferraro et al. 2001); Pal 13 (Clark et al. 2004); NGC 6752 (Sabbi et al. 2004); M 5 (Warren et al. 2006); and M 62 (Beccari et al. 2006), while in $\omega$ Cen the bimodality seems to be ruled out (Ferraro et al. 2006).

The bimodal distribution displays a minimum in the socalled zone of avoidance. The position and extension of the avoidance zone between the two main peaks seem to correlate with the cluster dynamical properties (mass segregation, dynamical evolution). Recently, Mapelli et al. (2006) proposed a model in which primordial and collisional BSS formation mechanisms are at work at the same time and have almost the same efficiency throughout the cluster life. In the proposed scenario, though, inner BSS would have a collisional origin, while outer BSS would have formed in the past from primordial binaries.

In the present paper, based on a sample of $56 \mathrm{GCs}$, we will look for any possible correlation between the number of BSS inside and outside the core and the half-mass radius and the GC main parameters. We describe in Sect. 2 the source of the quantities we use, while in Sect. 3 we show all the significant relations we find for our subsamples of BSS: Sect. 3.1 is focussed on the population of BSS inside and outside the core, while Sect. 3.2 analyses BSS selected inside and outside the half-mass radius. Section 3.3 shows the BSS luminosity functions. Section 4 presents a global analysis that makes use of the principal component analysis (PCA) technique to derive the overall dependencies between the relative number of BSS and the cluster properties. We finally discuss our results in Sect. 5.

\section{Quantities involved in this study}

The catalogue of BSS adopted for this study is basically the catalogue used by Piotto et al. (2004), which has been extracted from the HST snapshot catalogue described in Piotto et al. (2002). With respect to the BSS sample used by Piotto et al. (2004), we applied an additional selection on the faintest BSS as described at the end of the paper (see Appendix A), with the purpose of avoiding the inclusion of normal TO and lower subgiant branch stars with large photometric errors. We could reliably select BSS in a sample of 56 GCs. The difference in the number of BSS that we find with this new criterium and the one we adopted in Piotto et al. (2004) is on average 15\% for each cluster and does not show particular trends with the cluster parameters. The new selection does not have any significant impact, therefore, on the results presented elsewhere (Piotto et al. 2004; Davies et al. 2004), as it will be clarified in what follows.

For each GC we collected from the literature the most updated parameters. Some of them were calculated for the present work, as described in this section. In particular, our analysis makes use of the following quantities:

\section{Relative ages:}

We adopted the recent age estimate published by De Angeli et al. (2005) (48 GCs) and by Recio-Blanco et al. (2006) (8 GCs). Typical errors are of the order of 0.07 Gyr. Eight GCs have an age estimate with a larger error, namely NGC 5634, NGC 6093, NGC 6205, NGC 6356, NGC 6388, NGC 6624, NGC 6638, NGC 6864. For these clusters the average error is $\geq 1$ Gyr. We determined the relative ages applying the socalled vertical method on the HST snapshot CMDs and on the ground-based photometrically homogeneous CMD database by Rosenberg et al. (2000a,b).

\section{Metallicities:}

We used the two metallicity scales defined by Zinn \& West (1984) and Carretta \& Gratton (1997). These are the same metallicity scales used by De Angeli et al. (2005). We performed our analysis with both metallicity scales. Since we found no significant differences, we decided to publish results based on the Zinn \& West (1984) scale.

\section{Collisional parameter:}

The collisional parameter $\Gamma_{\text {coll }}$ was calculated as in Piotto et al. (2004), i.e., using the King (2002) formula to estimate the rate of stellar collisions per star and per year, assuming a mean $\mathrm{M} / \mathrm{L}$ ratio of 2 and a typical mass for the colliding stars of $0.4 M_{\odot}$.

\section{Central density:}

We calculated the central density $\rho_{\mathrm{c}}$ following the prescription given by Djorgovski (1993), using the new distance moduli determined by Recio-Blanco et al. (2005), and the central surface brightness given in the Harris (1996, February 2003 revision) catalogue.

\section{Maximum effective temperature of the horizontal branch:} The temperature of the hottest horizontal branch (HB) star $T_{\text {eff }}(\mathrm{HB})$ was extracted from Recio-Blanco et al. (2006). As in Recio-Blanco et al. (2006), in this paper $T_{\text {eff }}(\mathrm{HB})$ quantity is used as a signature of the horizontal branch (HB) extension.

\section{Cluster centres:}

We determined the positions of the cluster centres (Sbordone 2001) on the HST F439W images, using the mirrored autocorrelation method described in Djorgovski (1988). Before using this procedure, which is highly sensitive to the presence of clumps of bright stars, we subtracted the brightest sources from each image, and then applied a median filter. On the resulting images, we applied an algorithm that basically looks for photometric symmetries as signatures of the centre position has been applied. In fact, the auto-correlation function is evaluated on many equally-spaced portions of the image and then fitted with a paraboloid. The vertex of the paraboloid defines the cluster centre. The procedure is iterative; iterations are stopped when the variation in the $x$ and $y$ positions from one iteration to the next one are smaller than the error associated with the estimate of the centre position. This error is basically the error associated with the fit of the paraboloid in both dimensions. We give, in Table 1, the positions of the cluster centres in metachip coordinates for clusters where the procedure converged. The clusters not listed in Table 1 were too loose to allow a reliable measurement of the centre with the mirrored auto-correlation method. For these objects, we assumed the cluster centre was at the centre of the PC chip, i.e., $x_{\mathrm{c}}=600$ and $y_{\mathrm{c}}=1000$. 
Number of BSS and normalised number of BSS:

We will make use, in this paper, of two main quantities, i.e., the total number of BSS (selected in different regions) and the normalised number of BSS, which is the absolute number of BSS in a given region divided by the total luminosity coming from the stars in the same region (in unit of $10^{4} L_{\odot}$ ). Please note that, while one population of stars is usually selected and used as tracer (RGB, HB), here we used the whole population of evolved stars. The morphology of the evolved part of the CMD depends on the cluster parameters (age, metallicity just to mention the most important ones) and, therefore, normalising to one particular evolutionary sequence could possibly bias the analysis. Moreover, there are claims in the literature of radial gradients in the distribution of RGB stars or HB stars. To minimise these effects, we prefer to use the sampled luminosity as normalising factor. Our normalisation is, therefore, significantly different from the one applied by Leigh et al. (2007), as the latter normalise their BSS absolute number to different cluster populations (i.e., extremely hot HB stars, HB stars, RGB stars). Our normalisation factor should minimise the risk of contaminating the true dependence of the BSS population with the various cluster parameters with possible dependencies of the adopted normalising population on the same parameters.

The other cluster parameters (i.e., the core radius $r_{\mathrm{c}}$, the halfmass radius $r_{\mathrm{hm}}$, the core relaxation timescale $\tau_{\mathrm{rc}}$, the halfmass relaxation timescale $\tau_{\text {rhm }}$ ) are taken from the Harris (1996, February 2003 revision) catalogue. We calculated the total luminosity $M_{V}$ using the Harris integrated $V$ magnitude of the cluster and the distance modulus of Recio-Blanco et al. (2005), when available (otherwise, we used the distance modulus tabulated in the Harris catalogue). The central velocity dispersion, $\sigma_{\mathrm{v}}$, is taken from Pryor \& Meylan (1993).

Our sample of GCs is different from the one recently studied by Leigh et al. (2007). First of all, we include in our analysis NGC 6441 and NGC 6569, while NGC 6402, NGC 6522 and NGC 6712 have been excluded due to lower quality photometry and contaminated CMD. Most importantly, our selection has been made on the basis of our accurate error analysis, which stems from appropriate artificial star experiments (see Piotto et al. 2002, for more details). Clusters that have poor photometry have been excluded. Moreover, the results of the artificial star experiments allow us to correct the number of BSS according to the completeness level at each magnitude. We do not need to introduce, therefore, any cut in the photometric error of our BSS candidates. This makes our BSS catalogue significantly different from the one analysed by Leigh et al. (2007), who selected BSS from the CMDs of Piotto et al. (2002) assuming a cut based on a constant error (0.1 mag in both bands), while the photometric error strongly depends on the cluster density, and on the location of the BSS candidate with respect to the cluster centre ${ }^{1}$. For details on the BSS selection adopted in this work we refer the reader to the appendix (Appendix A). Our selection was performed in two steps: first, we identified BSS candidates by eye in the CMDs (a visual inspection of each diagram is particularly important to disentangle BSS candidate from HB stars); later on, we further selected BSS candidates by drawing a line approximately parallel to the sub-giant branch and at $0.7 \mathrm{mag}$ from

\footnotetext{
1 In addition, note that, in their selection, Leigh et al. (2007) used the photometric errors listed in the table in our Web pages. These errors, are the average fitting errors from DAOPHOT, which are an underestimate of the total error budget, and do not account for all of the effects of crowding as only the artificial star experiments can do.
}

the turnoff locations published by De Angeli et al. (2005). We recall here that for six clusters (namely NGC 6229, NGC 6293, NGC 6304, NGC 6441, NGC 6569, and NGC 6642) the position of the turnoff could not be determined using the same procedure adopted for the other clusters. In this case, the BSS have been visually selected from the CMD.

\section{Inner and outer samples of blue stragglers}

In this section, we present a few relevant monovariate relations between some cluster parameters and the BSS selected from different cluster regions:

- inside the core radius $\left(I_{\mathrm{c}} B S s\right)$;

- outside the core radius $\left(O_{\mathrm{c}} B S s\right)$;

- inside the half-mass radius $\left(I_{\mathrm{hm}} B S s\right)$;

- outside the half-mass radius $\left(O_{\mathrm{hm}} B S s\right)$.

In the following, we present and try to interpret the most relevant correlations.

\subsection{BSS populations inside and outside the cluster core}

In the analysis of the core population of BSS we did not take into account the PCC clusters, since for these objects the definition of core radius is not reliable (Trager et al. 1993, 1995). Therefore, we show monovariate relations for a subsample of 43 clusters, i.e., the whole sample of non-PCC clusters. This sample is different from the one studied by Leigh et al. (2007), who analysed together both normal and core-collapse clusters, claiming that they follow the same trend and that the percentage of PCC is negligible. In our sample, 13 out of 56 clusters are PCC (23\% of the global sample), a quite significant fraction.

The dependence on the total cluster luminosity is shown in Fig. 1. The upper panel shows the absolute number of $I_{\mathrm{c}} B S s$ and $O_{\mathrm{c}} B S s$. This plot can be directly compared with Fig. 5 of Mapelli et al. (2006) and Fig. 6 of Davies et al. (2004). When compared with Fig. 5 of Mapelli et al. (2006), the systematically smaller numbers of BSS in our plot are easily explained by taking into account the small HST field coverage and by the fact that we further subdivided our samples on the basis of the distance from the cluster centre.

In order to quantify the significance of our linear fits we performed a robust fit and calculated the dispersion using a bootstrap technique. In the insets of the figures illustrating the monovariate correlations, we give the best fitting straight line slope $\alpha$ together with its $1-\sigma$ error. Dashed and continuous lines fit the $I_{\mathrm{c}} B S s$ and $O_{\mathrm{c}} B S s$, respectively. NGC 4372 (with $M_{V}=-7.91$ ) has been excluded from the fit since it has just one BSS outside the cluster core, while clusters with 3 or more BSS in this region have been included, since the procedure is outlier resistant.

The vertical solid line marks the magnitude at which clusters produce approximately the same amount of primordial BSS and dynamical BSS, according to the predictions by Davies et al. (2004): less luminous clusters with $M_{V}>-8.8$ are expected to produce more BSS via mass transfer, while in clusters brighter than $M_{V}=-8.8$ collisions should play a more important role.

In order to establish whether or not the total luminosity (mass) has a prominent role in the population of BSS, we produced the same plot by normalising the number of BSS by the sampled luminosity. The lower panel of Fig. 1 shows that the normalised number of $I_{\mathrm{c}} B S s$ is on average higher than the normalised number of $O_{\mathrm{c}} B S s$, as expected from mass segregation, 
Table 1. Positions in metachip coordinates of cluster centres (Cols. 2 and 3 ), number of BSS, $I_{\mathrm{c}} B S s$ and $I_{\mathrm{hm}} B S s$ (Cols. 4 to 6) and normalising luminosities in the same regions in units of $10^{4}$ solar luminosities (Cols. 7 to 9 ).

\begin{tabular}{|c|c|c|c|c|c|c|c|c|}
\hline Name & $x_{\mathrm{c}}$ & $y_{\mathrm{c}}$ & $N_{\mathrm{BSS}}$, tot & $N_{\mathrm{BSS}}, r<r_{\mathrm{c}}$ & $N_{\mathrm{BSS}}, r<r_{\mathrm{hm}}$ & $L_{\text {tot }}$ & $L_{r<r_{\mathrm{c}}}$ & $L_{r<r_{\mathrm{hm}}}$ \\
\hline IC 4499 & 600.0 & 1000.0 & 33.0 & 21.0 & 33.0 & 1.682 & 0.942 & 1.471 \\
\hline NGC 0104 & 572.2 & 954.1 & 54.0 & 30.0 & 54.0 & 8.353 & 2.798 & 8.353 \\
\hline NGC 0362 & 610.5 & 955.3 & 69.0 & 37.0 & 64.0 & 6.770 & 1.809 & 5.359 \\
\hline NGC 1261 & 632.3 & 1041.0 & 68.0 & 43.0 & 53.0 & 4.655 & 1.643 & 2.847 \\
\hline NGC 1851 & 593.6 & 904.0 & 81.4 & 19.1 & 69.4 & 8.047 & 0.639 & 5.678 \\
\hline NGC 1904 & 623.7 & 906.1 & 39.0 & 19.0 & 33.0 & 4.187 & 0.866 & 3.346 \\
\hline NGC 2808 & 598.3 & 942.9 & 107.8 & 46.6 & 86.8 & 18.809 & 5.332 & 12.939 \\
\hline NGC 3201 & 600.0 & 1000.0 & 18.0 & 18.0 & 18.0 & 0.782 & 0.678 & 0.782 \\
\hline NGC 4147 & 598.5 & 973.1 & 28.0 & 12.0 & 26.0 & 1.213 & 0.211 & 0.804 \\
\hline NGC 4372 & 600.0 & 1000.0 & 11.0 & 10.0 & 11.0 & 0.586 & 0.561 & 0.586 \\
\hline NGC 4590 & 600.0 & 1000.0 & 24.0 & 15.0 & 24.0 & 1.314 & 0.606 & 1.260 \\
\hline NGC 4833 & 600.0 & 1000.0 & 30.0 & 19.0 & 30.0 & 2.156 & 1.307 & 2.156 \\
\hline NGC 5024 & 549.1 & 918.3 & 58.0 & 30.0 & 51.0 & 6.635 & 2.456 & 5.604 \\
\hline NGC 5634 & 553.4 & 946.2 & 46.0 & 29.0 & 37.0 & 4.638 & 1.422 & 3.027 \\
\hline NGC 5694 & 594.7 & 994.5 & 42.5 & 16.3 & 33.5 & 6.005 & 0.922 & 4.225 \\
\hline NGC 5824 & 611.5 & 956.2 & 98.7 & 23.0 & 74.7 & 15.665 & 1.719 & 10.973 \\
\hline NGC 5904 & 600.0 & 1000.0 & 28.0 & 19.0 & 28.0 & 4.710 & 1.599 & 4.710 \\
\hline NGC 5927 & 540.9 & 979.5 & 23.0 & 23.0 & 23.0 & 4.035 & 0.921 & 2.920 \\
\hline NGC 5946 & 558.2 & 986.5 & 14.0 & 2.0 & 11.0 & 4.805 & 0.328 & 2.426 \\
\hline NGC 5986 & 600.0 & 1000.0 & 49.6 & 31.5 & 48.6 & 7.438 & 3.206 & 5.833 \\
\hline NGC 6093 & 590.5 & 1001.3 & 44.3 & 20.1 & 40.3 & 9.251 & 1.861 & 5.905 \\
\hline NGC 6171 & 600.0 & 1000.0 & 33.0 & 17.0 & 33.0 & 0.981 & 0.358 & 0.981 \\
\hline NGC 6205 & 600.0 & 1000.0 & 18.0 & 8.0 & 17.0 & 5.520 & 2.566 & 5.024 \\
\hline NCG 6218 & 600.0 & 1000.0 & 31.0 & 17.0 & 31.0 & 1.113 & 0.379 & 1.113 \\
\hline NGC 6229 & 564.4 & 951.3 & 79.7 & 38.3 & 67.7 & 8.285 & 2.108 & 5.368 \\
\hline NGC 6235 & 600.0 & 1000.0 & 18.0 & 8.0 & 15.0 & 1.717 & 0.485 & 1.109 \\
\hline NGC 6266 & 615.7 & 936.6 & 49.1 & 18.0 & 43.1 & 11.633 & 1.978 & 10.495 \\
\hline NGC 6273 & 576.5 & 926.7 & 53.1 & 19.0 & 47.1 & 12.003 & 4.041 & 10.047 \\
\hline NGC 6284 & 556.9 & 984.2 & 22.0 & 3.0 & 17.0 & 4.915 & 0.321 & 3.230 \\
\hline NGC 6287 & 575.2 & 970.6 & 13.0 & 9.0 & 13.0 & 2.723 & 0.621 & 1.647 \\
\hline NGC 6293 & 564.6 & 923.4 & 35.1 & 3.1 & 25.1 & 4.628 & 0.193 & 3.578 \\
\hline NGC 6304 & 603.4 & 996.9 & 53.0 & 20.0 & 50.0 & 3.223 & 0.625 & 2.841 \\
\hline NGC 6342 & 569.6 & 991.1 & 30.1 & 7.0 & 22.1 & 1.443 & 0.049 & 0.966 \\
\hline NGC 6356 & 576.1 & 980.7 & 72.4 & 30.9 & 47.3 & 9.006 & 2.372 & 5.660 \\
\hline NGC 6362 & 600.0 & 1000.0 & 25.0 & 22.0 & 25.0 & 1.054 & 0.752 & 1.054 \\
\hline NGC 6388 & 606.4 & 962.2 & 133.2 & 42.5 & 105.1 & 27.536 & 4.189 & 19.180 \\
\hline NGC 6397 & 600.0 & 1000.0 & 11.0 & 2.0 & 11.0 & 0.384 & 0.005 & 0.384 \\
\hline NGC 6441 & 557.5 & 965.0 & 143.0 & 48.9 & 109.7 & 31.433 & 3.912 & 20.394 \\
\hline NGC 6544 & 600.0 & 1000.0 & 20.0 & 1.0 & 19.0 & 0.912 & 0.012 & 0.907 \\
\hline NGC 6569 & 600.0 & 1000.0 & 52.7 & 26.6 & 46.7 & 6.742 & 2.020 & 5.842 \\
\hline NGC 6584 & 600.0 & 1000.0 & 31.0 & 21.0 & 26.0 & 2.396 & 1.196 & 1.496 \\
\hline NGC 6624 & 572.1 & 963.7 & 45.0 & 11.0 & 34.0 & 2.936 & 0.205 & 2.160 \\
\hline NGC 6637 & 558.0 & 946.2 & 48.0 & 29.0 & 40.0 & 3.240 & 1.123 & 2.271 \\
\hline NGC 6638 & 599.8 & 990.0 & 45.0 & 22.0 & 36.0 & 4.470 & 1.586 & 2.718 \\
\hline NGC 6642 & 560.8 & 942.2 & 41.0 & 16.0 & 34.0 & 2.504 & 0.339 & 1.608 \\
\hline NGC 6652 & 581.2 & 973.4 & 45.0 & 20.0 & 36.0 & 1.542 & 0.144 & 1.069 \\
\hline NGC 6681 & 600.0 & 1000.0 & 16.0 & 0.0 & 16.0 & 2.267 & 0.020 & 1.761 \\
\hline NGC 6717 & 600.0 & 1000.0 & 33.0 & 5.0 & 30.0 & 0.636 & 0.033 & 0.392 \\
\hline NGC 6723 & 600.0 & 1000.0 & 24.0 & 16.0 & 23.0 & 2.268 & 1.429 & 2.188 \\
\hline NGC 6838 & 600.0 & 1000.0 & 28.0 & 9.0 & 27.0 & 0.386 & 0.142 & 0.374 \\
\hline NGC 6864 & 600.0 & 1000.0 & 58.6 & 26.4 & 58.6 & 10.877 & 1.819 & 7.422 \\
\hline NGC 6934 & 635.7 & 951.4 & 24.0 & 19.0 & 23.0 & 4.077 & 1.462 & 2.650 \\
\hline NGC 6981 & 600.0 & 1000.0 & 28.0 & 16.0 & 22.0 & 2.062 & 0.987 & 1.421 \\
\hline NGC 7078 & 601.2 & 981.8 & 46.0 & 7.0 & 42.0 & 10.503 & 0.612 & 8.901 \\
\hline NGC 7089 & 600.0 & 1000.0 & 15.0 & 8.0 & 13.0 & 4.733 & 1.307 & 3.085 \\
\hline NGC 7099 & 633.6 & 997.1 & 35.0 & 13.0 & 33.0 & 1.766 & 0.104 & 1.495 \\
\hline
\end{tabular}

being BSS more massive than normal cluster members (this effect will be present in all of the following figures). Interestingly enough, the slopes of the two straight lines best fitting the $I_{\mathrm{c}} B S S$ and $O_{\mathrm{c}} B S S$ samples are the same, within the fitting errors. Accounting for the error bars, for the $O_{\mathrm{c}} B S s$ subsample, all clusters follow the same relation with the possible exception of
NGC 6838 . The same is true for the $I_{\mathrm{c}} B S s$, with the only possible exceptions of NGC 6652, NGC 5927, and NGC 6864.

Figure 1 further demonstrates that the normalised number of BSS per unit luminosity nicely anticorrelates with the cluster luminosity. This anticorrelation is similar for both the $I_{\mathrm{c}} B S s$ and the $O_{\mathrm{c}} B S s$. This implies that the frequency of BSS, 

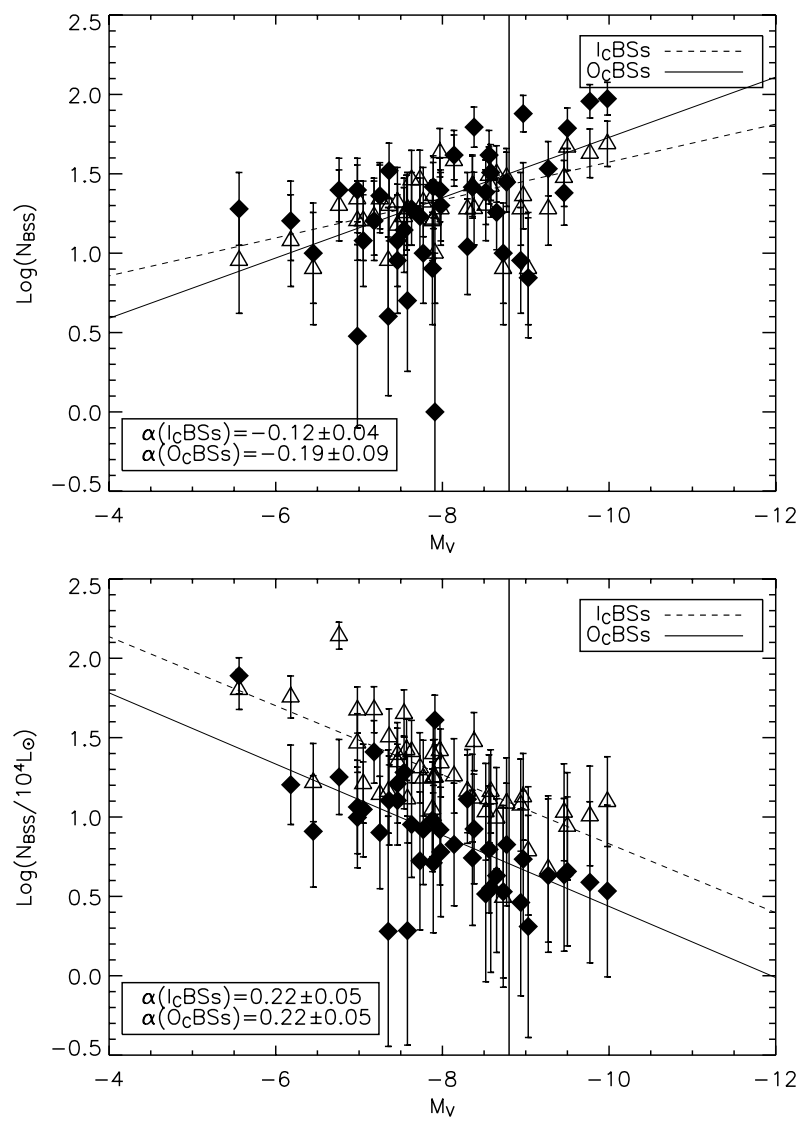

Fig. 1. Upper panel: total number of BSS as a function of the host cluster integrated absolute magnitude. Diamonds refer to the number of $O_{\mathrm{c}} B S s$, triangles to the number of $I_{\mathrm{c}} B S s$. Lower panel: same plot with the number of BSS normalised to the sampled luminosity in unit of $10^{4}$ solar luminosities in the $F 555 W$ HST band.

independent from where it is measured, holds the same dependence on the cluster total light (mass). The relation of the relative number of $O_{\mathrm{c}} B S s$ vs. the integrated luminosity of the host cluster is presented here for the first time (in Piotto et al. 2004, the two samples were mixed together).

For both $I_{\mathrm{c}} B S s$ and $O_{\mathrm{c}} B S s$ the relation is steep and statistically significant (at a five $\sigma$ level for both samples), thus confirming that the total mass of a cluster is important in determining the properties of the BSS population. We will demonstrate, in fact, that the total luminosity is the predominant parameter in setting the BSS population in a GC. This result is in agreement with what previously found by Piotto et al. (2004) and Leigh et al. (2007). The upper panel of Fig. 2 shows the total number of $I_{\mathrm{c}} B S s$ and $O_{\mathrm{c}} B S s$ plotted as a function of the central density: the $I_{\mathrm{c}} B S s$ show a small dependence on the central density, consistent with a flat relation, while the $O_{\mathrm{c}} B S s$ show a more significant correlation with this parameter. This is still true if we exclude from the fit the clusters with one to three BSS detected outside the core radius (NGC 4372 and NGC 6362, respectively).

The lower panel of Fig. 2 shows the same relation with normalised quantities. The two slopes, as well as the scatters, tend to be very similar. For the $I_{\mathrm{c}} B S s$ however, the error on the slope is such that a null dependence on the central density can not be completely ruled out. Thus, the central density does not seem to play a major role in determining the properties of $I_{\mathrm{c}} B S s$. For the $O_{\mathrm{c}} B S s$ the dependence on the central density is similar and the error on the slope of the fitted relation confirms (at about three $\sigma$ level) that there is a correlation.
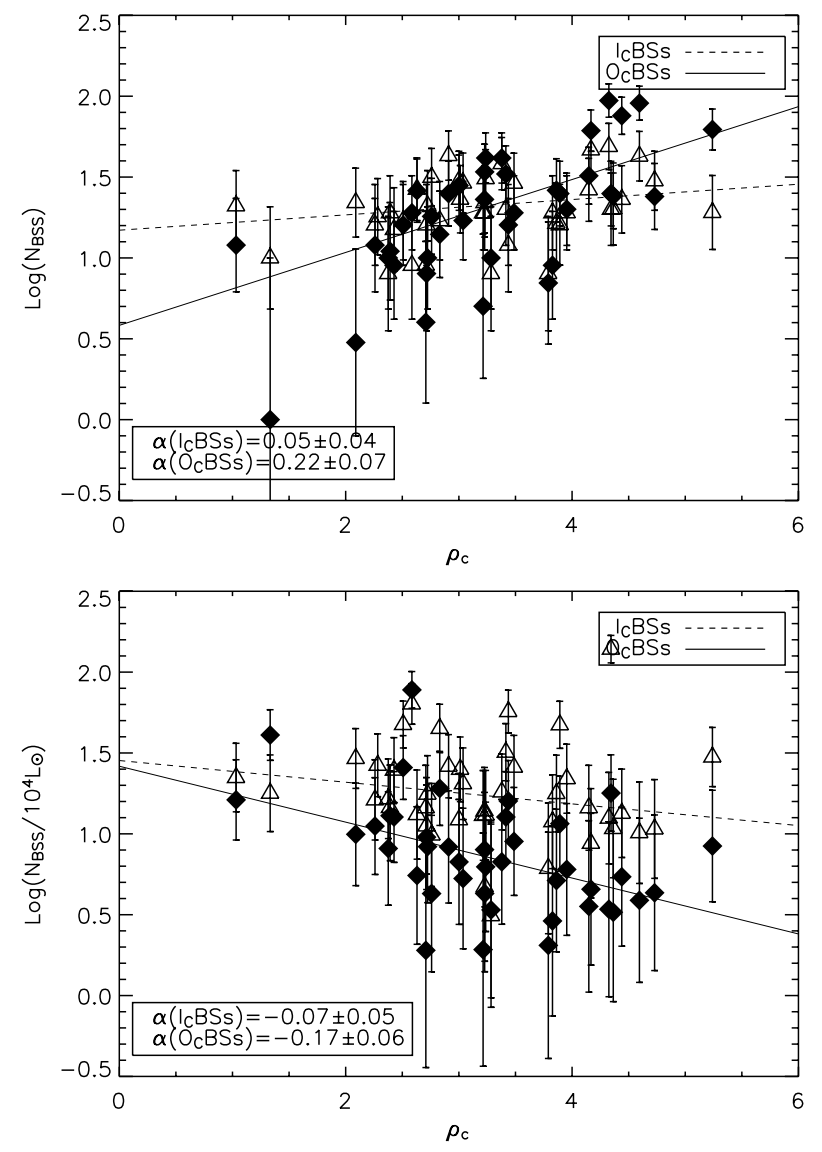

Fig. 2. Upper panel: total number of BSS as a function of the host cluster central density. Diamonds and triangles refer to the number of $O_{\mathrm{c}} B S s$ and $I_{\mathrm{c}} B S s$ respectively. Lower panel: relative number of BSS (normalised to the sampled luminosity in unit of $10^{4}$ solar luminosities in the F555W HST band) vs. central density.

The relative number of BSS is on average decreasing at increasing $\rho_{\mathrm{c}}$. Could this reflect the fact that primordial BSS evolve more rapidly in clusters having higher central density? The anti-correlation with the central density is stronger in the external regions, and this is more difficult to understand. Also, the large dispersion of the $I_{\mathrm{c}} B S s$ relative frequency as a function of $\rho_{\mathrm{c}}$ is an additional empirical evidence that needs to be interpreted.

In principle, the BSS population should be strongly influenced by the dynamical evolutionary timescale of the host cluster. This should be reflected by the relations shown in Fig. 3. However, in the upper panel the normalised number of $I_{\mathrm{c}} B S s$ very marginally anticorrelates with the central relaxation timescale $\tau_{\mathrm{rc}}$, while the normalised number of $O_{\mathrm{c}} B S s$ bears no significant dependence on this parameter. The lower panel shows a more significant (but still at $\sim 2 \sigma$ level) anticorrelation of the normalised number of $I_{\mathrm{c}} B S s$ with the half-mass relaxation timescale.

Therefore, there is some indication of an anticorrelation of the normalised number of BSS with both the core and the halfmass relaxation timescales, but only for the $I_{\mathrm{c}} B S s$, and this dependence is more significant when using the half-mass relaxation time. Also in this case, we note a large dispersion. This observational evidence is likely the consequence of the fact that in the outskirts of the clusters the relaxation timescale is larger than the Hubble time. We will see in Sect. 4 that the relaxation 

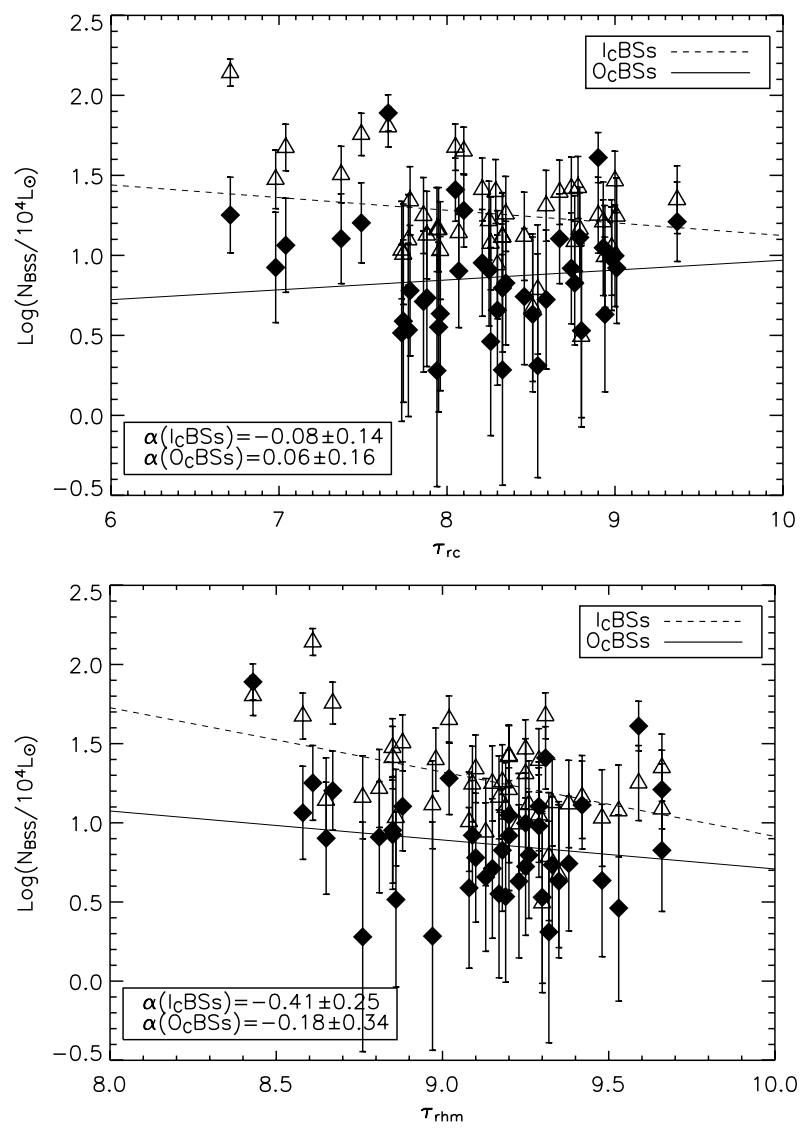

Fig. 3. Relative number of BSS as a function of the host cluster relaxation times: the upper panel refers to the half-mass relaxation time, the lower panel to the core relaxation time. Diamonds and triangles show the frequencies of $O_{\mathrm{c}} B S s$ and $I_{\mathrm{c}} B S s$ respectively. The number of BSS is normalised to the sampled luminosity in unit of $10^{4}$ solar luminosities in the $F 555 W$ HST band.

times have, in fact, some influence on the global properties of BSS populations.

Finally, we show four other monovariate relations for the normalised numbers of $I_{\mathrm{c}} B S s$ and $O_{\mathrm{c}} B S s$, namely with the cluster metallicity, the maximum temperature of $\mathrm{HB}$, the central velocity dispersion (for clusters that have this measurement), and the collisional parameter $\Gamma_{\text {coll }}$. The upper left panel of Fig. 4 shows that there is no dependence between the normalised number of $I_{\mathrm{c}} B S s$ and $O_{\mathrm{c}} B S s$ and the cluster metallicity. Instead, there is a correlation between the maximum temperature of the $\mathrm{HB}$ and the relative number of BSS (Fig. 4, upper right panel), with hotter HB clusters (i.e. clusters with more extended HBs) hosting less BSS per unit luminosity, both inside and outside the core. A possible correlation is also found between the frequency of $I_{\mathrm{c}} B S s$ and $O_{\mathrm{c}} B S s$ and the central velocity dispersion of the host clusters. Clusters with higher central velocity dispersion host less BSS per unit luminosity with respect to clusters with low $\sigma_{\mathrm{v}}$. This last relation, in agreement with what Leigh et al. (2007) found, was somewhat expected, given the existing tight relation between the cluster luminosity and the central velocity dispersion (Djorgovski 1991), which is analogous to the Faber-Jackson relation for elliptical galaxies. Because the maximum temperature of the HB correlates with the total cluster magnitude (Recio-Blanco et al. 2006), it is not clear whether the correlation of Fig. 4 (upper right panel) is just the consequence of the former correlation.
Though the measurement of the central velocity dispersion is available only for a small subsample of clusters, we have investigated the possible relation between the number of BSS and the collisional parameter $\Gamma_{\text {coll }}$ (see Fig. 4). There is some indication for a possible anticorrelation, but the statical significance is very low, particularly for the $I_{\mathrm{c}} B S s$ sample.

\subsection{Half-mass radius population}

We discuss, in this section, monovariate relations involving BSS that lie inside and outside the half-mass radius $\left(I_{\mathrm{hm}} B S s\right.$ and $O_{\mathrm{hm}} B S s$ respectively). In this case, we include in the analysis the PCC clusters (plotted with larger open symbols surrounding the usual ones). First, we show the same plots commented on in the previous section, showing the relations between the total and relative numbers of BSS and the total cluster luminosity, the central density, and the relaxation times. Only clusters having at least one BSS candidate outside the half-mass radius are included in the plots, this reduces our sample to 40 clusters. Among the remaining clusters, the percentage of $O_{\mathrm{hm}} B S s$ on the total number of BSS ranges from $\sim 2 \%$ for NGC 6838 to $\sim 34 \%$ for NGC 6356. The lower panel in Fig. 5, like in Fig. 1, shows a very significant relation of the BSS frequency with the cluster integrated magnitude. The large separation between the $I_{\mathrm{hm}} B S s$ and $O_{\mathrm{hm}} B S s$ total number in the upper panel is due to the effects of mass segregation and to the fact that only a small part of the region outside the half-mass radius is sampled by the HST images.

Figures 5 to 7 confirm the trends discussed in Sect. 3.1. We notice however, that differences in the trends between the inner and outer BSS absolute numbers as a function of the various parameters $\left(M_{V}, \rho_{\mathrm{c}}, \tau_{\mathrm{rc}}\right)$ tend to become less evident when we divide the BSS samples using $r_{\mathrm{hm}}$ instead of $r_{\mathrm{c}}$ as cut-off value.

In any case, there seems to be a marginal anticorrelation of the $I_{\mathrm{hm}} B S s$ frequency with $\tau_{\mathrm{rhm}}$. Also, the $O_{\mathrm{hm}} B S s$ tend to anticorrelate with the half-mass relaxation time, but the relation is not statistically significant. We note a larger dispersion in the frequency of the $O_{\mathrm{hm}} B S s$ when plotted against $\tau_{\mathrm{rhm}}$ than in the analogous plot for the $I_{\mathrm{hm}} B S s$ sample.

We do not see any significant difference in the trends defined by normal King-model clusters and PCC clusters.

\subsection{Luminosity functions}

We also considered the possible effects of $M_{V}, \rho_{\mathrm{c}}, \tau_{\mathrm{rc}}$ on the BSS luminosity function (LF). As in Piotto et al. (2004), for each BSS we calculated the luminosity relative to the parent cluster TO luminosity, extracted from De Angeli et al. (2005). We then divided the catalogue of GCs into two samples according to 1$)$ their luminosity $\left(M_{V}>-8.8\right.$ and $\left.M_{V} \leq-8.8\right)$; 2) their central density $\left(\rho_{\mathrm{c}}>3.5\right.$ and $\left.\left.\rho_{\mathrm{c}} \leq 3.5\right) ; 3\right)$ their core relaxation timescale $\left(\tau_{\mathrm{rc}}>8.0\right.$ and $\left.\tau_{\mathrm{rc}} \leq 8.0\right)$. We chose to divide the sample in clusters that are more luminous and less luminous than $M_{V}=-8.8$ because this was the value also used in Piotto et al. (2004) and Davies et al. (2004) (see these papers for a discussion on the origin of this particular value). As for the cuts in $\rho_{\mathrm{c}}$ and $\tau_{\text {rc }}$, we simply used the values that, more or less, split the whole sample in two equal parts. We also performed our calculations for slightly different values ( $\rho_{\mathrm{c}}$ from 3 to 3.7 and $\tau_{\mathrm{rc}}$ from 7.8 to 8.2 ), but the results do not change. Using very different values would deteriorate the statistics for one of the samples.

In our first analysis, we excluded the PCC clusters. The LFs of each cluster have been added together to construct the total 

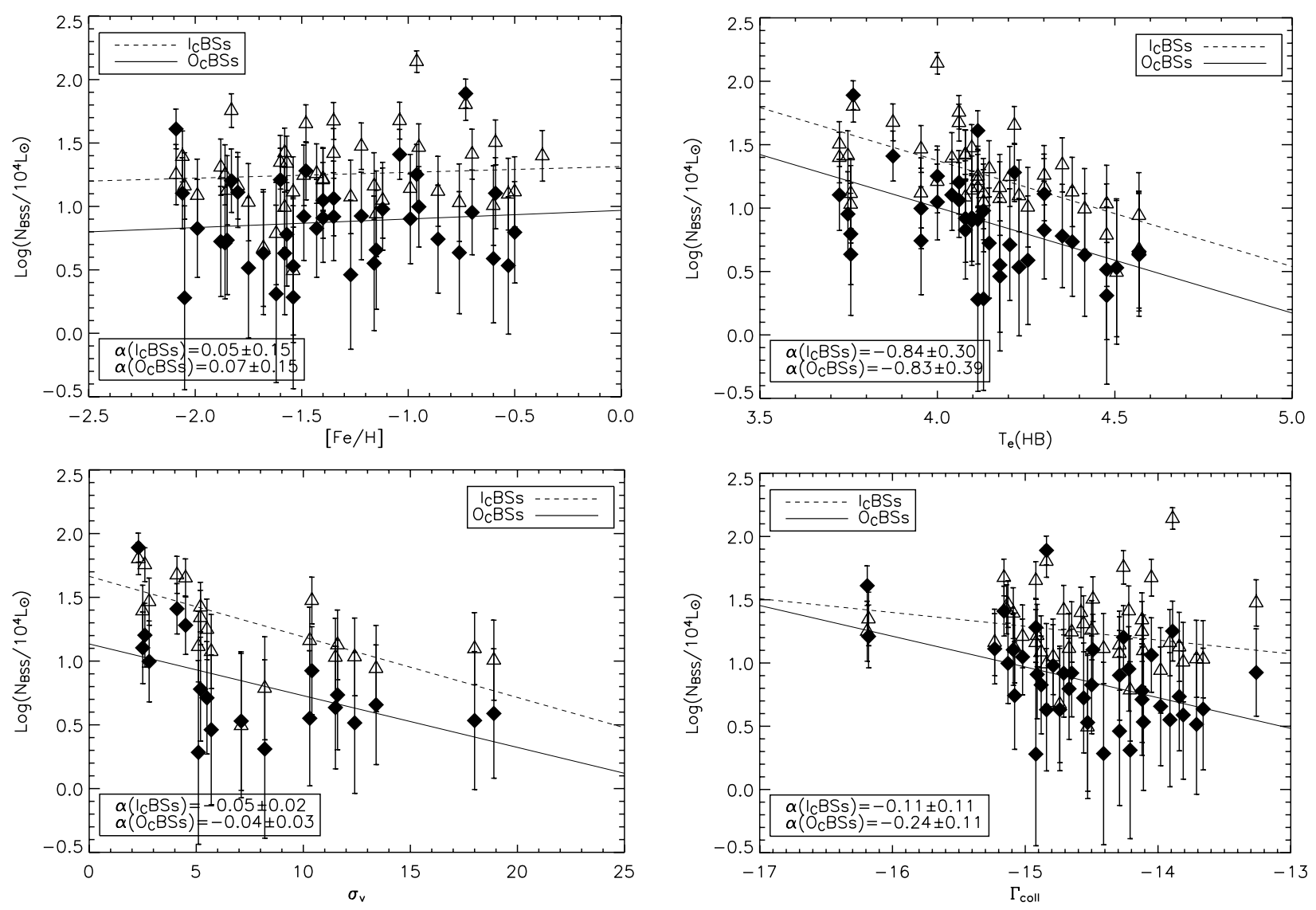

Fig. 4. Upper left panel: normalised number of BSS as a function of the host cluster metallicity. Diamonds refer to the number of $O_{\mathrm{c}} B S s$ triangles to the number of $I_{\mathrm{c}} B S$. Upper right panel: normalised number of BSS as a function of the host cluster maximum HB temperature. Lower left panel: normalised number of $I_{\mathrm{c}} B S s$ and $O_{\mathrm{c}} B S s$ as a function of the central velocity dispersion. Lower right panel: normalised number of $I_{\mathrm{c}} B S s$ and $O_{\mathrm{c}} B S s$ as a function of the collisional parameter $\Gamma_{\text {coll }}$.

LF for each subsample. The final luminosity functions have been normalised to the total number of BSS in each subsample.

Figure 8 shows the three sets of normalised luminosity functions we obtain by subdividing our subsample of non-PCC clusters (43 objects) according to their $M_{V}, \rho_{\mathrm{c}}, \tau_{\text {rc }}$ (from top to bottom in the figure). In each panel, the lower subpanels refer to the $O_{\mathrm{c}} B S s$, the upper subpanels to the $I_{\mathrm{c}} B S s$. The continuous line corresponds to BSS in clusters with $M_{V}>-8.8, \rho>3.5$ and $\tau_{\mathrm{rc}}>8.0$, while the dashed line corresponds to BSS in clusters with $M_{V} \leq-8.8, \rho \leq 3.5$ and $\tau_{\text {rc }} \leq 8.0$.

Figure 8 shows that the BSS peak tends to be brighter in more luminous (massive) clusters, in clusters with higher central densities, and in clusters with smaller $\tau_{\text {rc }}$. This holds for both subsamples of inner and outer core BSS.

Apparently, as already discussed by Piotto et al. (2004), the LFs coming from the different subgroups are different. However, it is difficult to assess the statistical significance of this difference. Also, a Kolmogorov-Smirnov test is not conclusive, as it provides between $50 \%$ and $90 \%$ probability (depending on the adopted LF binning) that the two LFs in each of the panels of Fig. 8 (and the consecutive figures showing luminosity functions) follow the same distribution.

These differences are clearly present, and go in the same direction, when we select the BSS inside and outside the half-mass radius (see Sect. 3.2), as shown in Fig. 9.

Figure 10, shows the luminosity functions of our subsamples not divided according to their distance from the centre of the cluster. In this figure, we include all the clusters, including PCC ones. The trends already shown by the subpopulations of $I_{\mathrm{c}} B S s$,
$O_{\mathrm{c}} B S s, I_{\mathrm{hm}} B S s$ and $O_{\mathrm{hm}} B S s$ are statistically more significant, due to the larger sample of BSS. If we exclude the PCC clusters, the general trend of the LFs is not changing (Fig. 11).

These results confirm what Piotto et al. (2004) previously found, and are not in agreement with the conclusions of Leigh et al. (2007), who do not find any significant difference between luminosity functions of subsamples having different cluster magnitudes. It is conceivable that the different selection criteria and the impossibility by Leigh et al. (2007) to correct the LFs by incompleteness effects have altered their results.

We checked that PCC clusters display luminosity functions very similar to the ones of normal, King-model profile clusters when selected according to their integrated luminosity, i.e., BSS are on average brighter in more massive clusters (though the LFs are more noisy).

Finally, we did check whether the luminosity functions of subsamples of clusters having different metallicities were different. In particular, we divided the sample into clusters with low and high metallicities, using as a cut value $[\mathrm{Fe} / \mathrm{H}]=-1.25$. Again, we chose this value to split the sample into subsamples of the same size. We find that more metal-rich clusters seem to host slightly brighter BSS (see Fig. 12).

\section{Multivariate analysis}

As seen in previous sections, the monovariate analysis of different populations of blue stragglers does not always have a straightforward interpretation. This is a consequence of the fact 

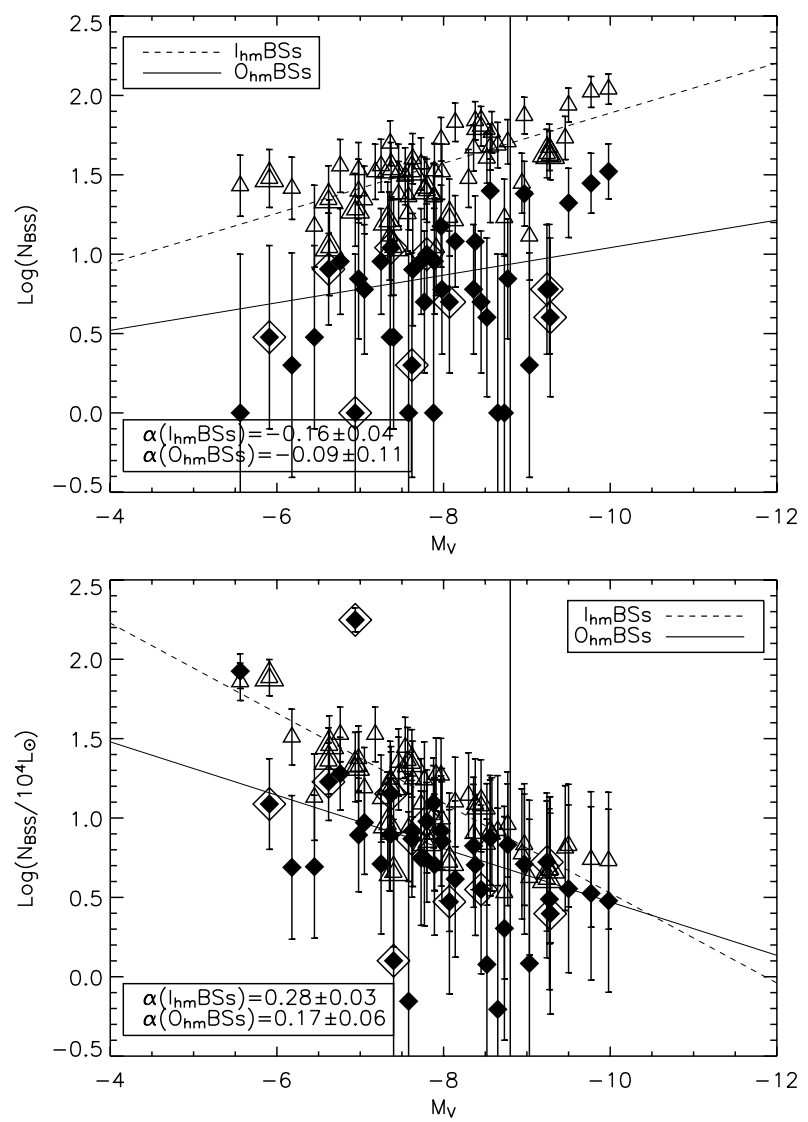

Fig. 5. Upper panel: total number of BSS as a function of the host cluster integrated absolute magnitude. Diamonds and triangles refer to the number of $O_{\mathrm{hm}} B S s$ and $I_{\mathrm{hm}} B S s$ respectively. Empty symbols mark PCC clusters. The linear fits is calculated excluding these last clusters. Lower panel: same plot with the relative number of BSS, normalised to the sampled luminosity.

that GCs constitute a complex family of objects, which depend on many parameters, often mutually related (Djorgovski \& Meylan 1994, and references therein). A more detailed analysis of the BSS populations has to take into account all the possible cluster parameters using a multivariate approach. The multivariate analysis tool has been used in the past to infer correlations among various properties of the Galactic globular cluster system (Brosche \& Lentes 1984; Djorgovski 1991; Bellazzini et al. 1992; Djorgovski et al. 1993; Djorgovski \& Meylan 1994; Recio-Blanco et al. 2006).

The basic concept of a multivariate analysis is quite simple: first, one has to define the correlation matrix involving the whole space of parameters, and then look for the best combination of the initial vectors that accounts for most of the variance. In defining the correlation matrix, the entries need to be normalised, i.e. the values must have null mean and unit variance. This ensures that the analysis is not biased by the different units in which the parameters are expressed. The second step is to produce a set of eigenvectors and corresponding eigenvalues that helps in restricting the number of variables used to reproduce most of the initial variance. Usually, eigenvectors with eigenvalue larger than 1 are considered significant, while the others bear lower weight. The composition of the first eigenvectors in terms of the original parameters determines what parameters predominantly influence the initial sample.

We performed the principal component analysis on our subsamples of clusters to determine how the normalised (and the
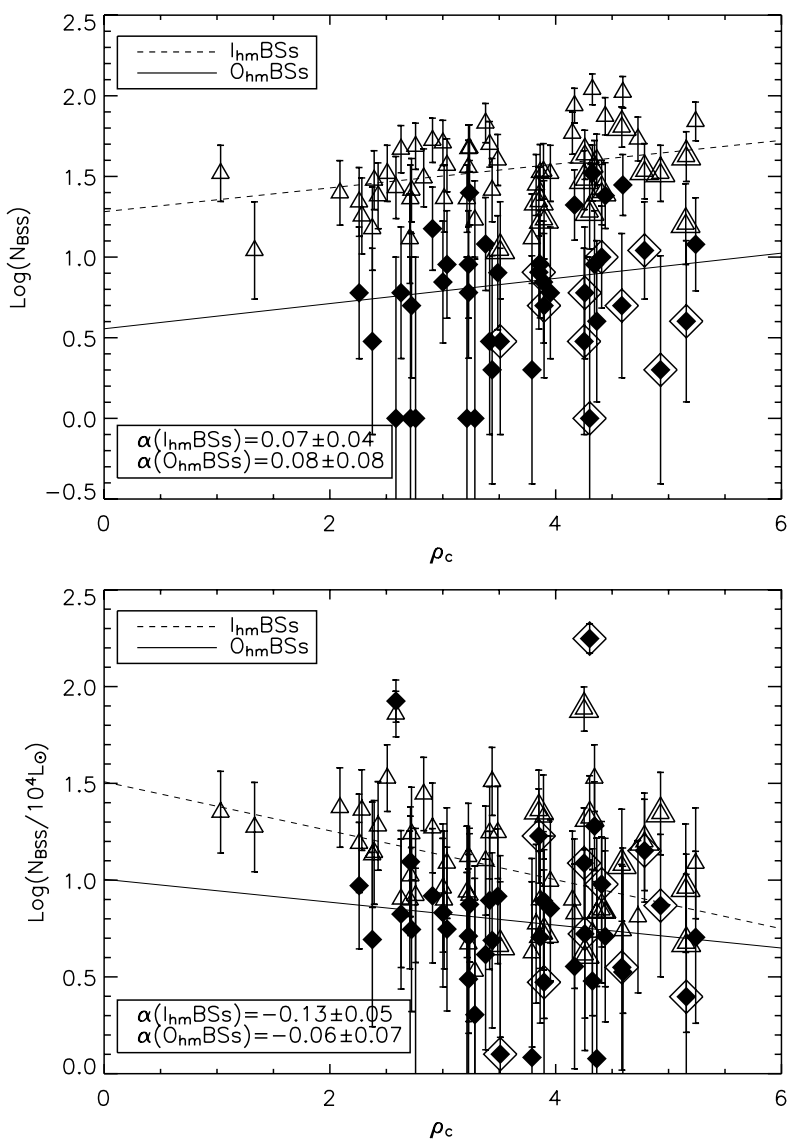

Fig. 6. Upper panel: total number of BSS as a function of the host cluster central density. Diamonds and triangles refer to the number of $O_{\mathrm{hm}} B S s$ and $I_{\mathrm{hm}} B S s$ respectively. Empty symbols mark PCC clusters. The linear fits are calculated excluding these last clusters. Lower panel: same plot with the relative number of BSS normalised to the sampled luminosity.

not normalised) number of BSS is influenced by the various parameters: in our first analysis we included $M_{V}, \Gamma_{\text {coll }}, \tau_{\mathrm{rc}}, \tau_{\mathrm{rhm}}, \rho_{\mathrm{c}}$, $[\mathrm{Fe} / \mathrm{H}]$ (in the ZW scale), Age, $T_{\text {eff }}(\mathrm{HB})$ and $\sigma_{\mathrm{v}}$. Data were first normalised to have null mean and unit variance. We analysed data for 29 GCs, i.e., the same 56 GCs in Piotto et al. (2004), but excluded the ones with one of the entering parameters missing.

As an example, in Table 2 we show the correlation matrix involving the whole population of BSS: the major contributions to the total variance arise from the cluster luminosity, the maximum temperature along the $\mathrm{HB}$ (this last correlated with the cluster luminosity, as demonstrated in Recio-Blanco et al. 2006), the velocity dispersion, and the half-mass relaxation time. The collisional parameter $\Gamma_{\text {coll }}$ also plays a role in determining the properties of the global population of BSS. This basically confirms the trends observed in the monovariate analysis.

The second part of Table 2 refers to the same analysis performed on the same sample, but without normalising the number of BSS to the luminosity. It shows that the main contributors to the total variance are basically the same, though they have often a different sign. This is a consequence of the fact that more luminous clusters, in general have more BSS, but a lower specific frequency. The contribution of the luminosity and the maximum temperature along the $\mathrm{HB}$ is lower, while the central velocity dispersion becomes more important.

Table 3 lists the eigenvalues and cumulative percentages of the derived eigenvectors and demonstrates that, while the 

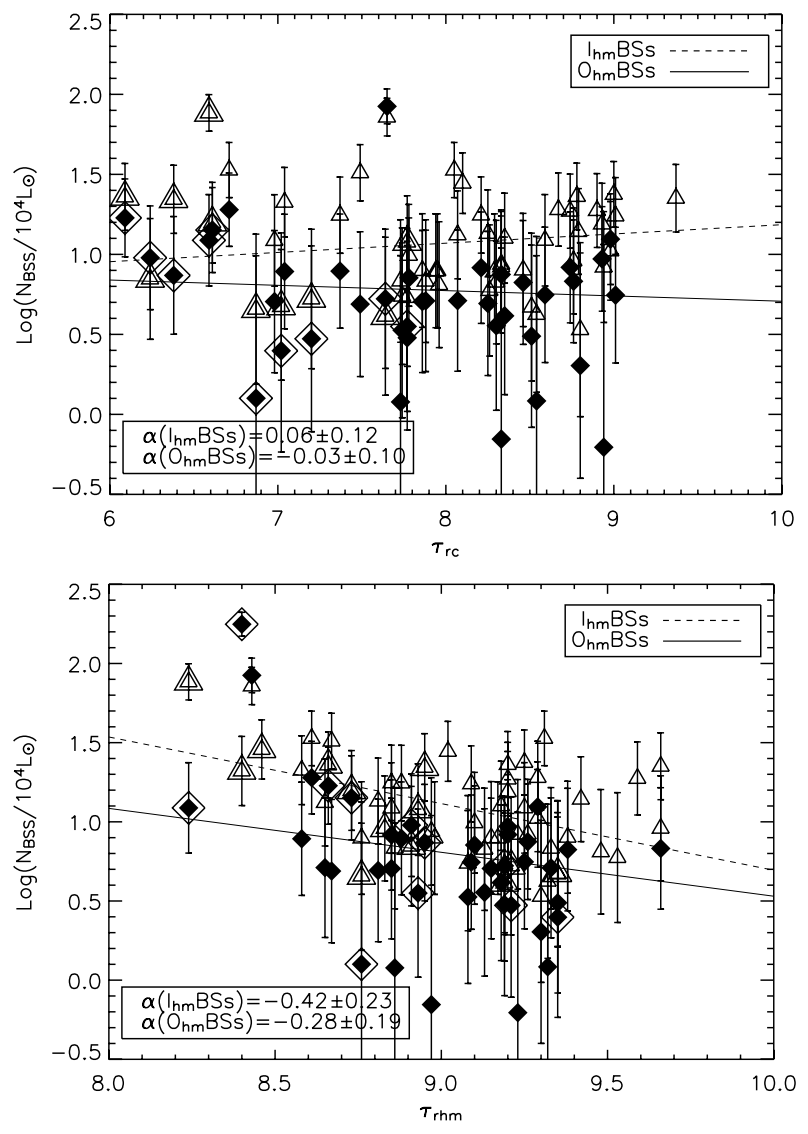

Fig. 7. Normalised number of BSS as a function of the host cluster relaxation times: the upper panel refers to the half-mass relaxation time, the lower panel to the core relaxation time. Diamonds and triangles refer to the number of $O_{\mathrm{hm}} B S s$ and $I_{\mathrm{hm}} B S s$ respectively. Empty symbols mark PCC clusters. The linear fits are calculated excluding these last clusters.

primary influence of the above parameters is clear, the statistical dimension of the analysed space parameter is larger than 3 . The first three eigenvectors, in fact, can barely account for $81 \%$ of the total variance, and at least six of them have to be considered to account for $95 \%$ of it.

A better way to visualise how the various parameters influence the total variance is to look at the the projection of the initial vectors on the first two eigenvectors (see Fig. 13). The parameters that most influence the BSS relative number (here shown by the thick vector) are those represented by the vectors that run parallel (or anti-parallel) to the BSS number vector: the longer the projection of vector on the $N_{\mathrm{BSS}}$ vector, the higher its influence to the total variance.

The frequency of BSS in our sample of clusters depends, thus, strongly on the total luminosity, on the maximum temperature along the $\mathrm{HB}$, on the velocity dispersion, and on the half-mass relaxation timescale, (vectors large and not orthogonal in Fig. 13). This can be seen both in the upper panel, which shows the projection on the plane of the first two eigenvectors, and in the lower panel, which is the projection on the plane of the first and third eigenvector. As already seen in the monovariate correlation, the effect of the central density $\rho_{\mathrm{c}}$ emerges from this second projection plane, together with the collisional parameter $\Gamma_{\text {coll }}$, though this effect is less important than the correlation with the other above mentioned parameters. The relative age of GCs clearly has no influence, probably because the age
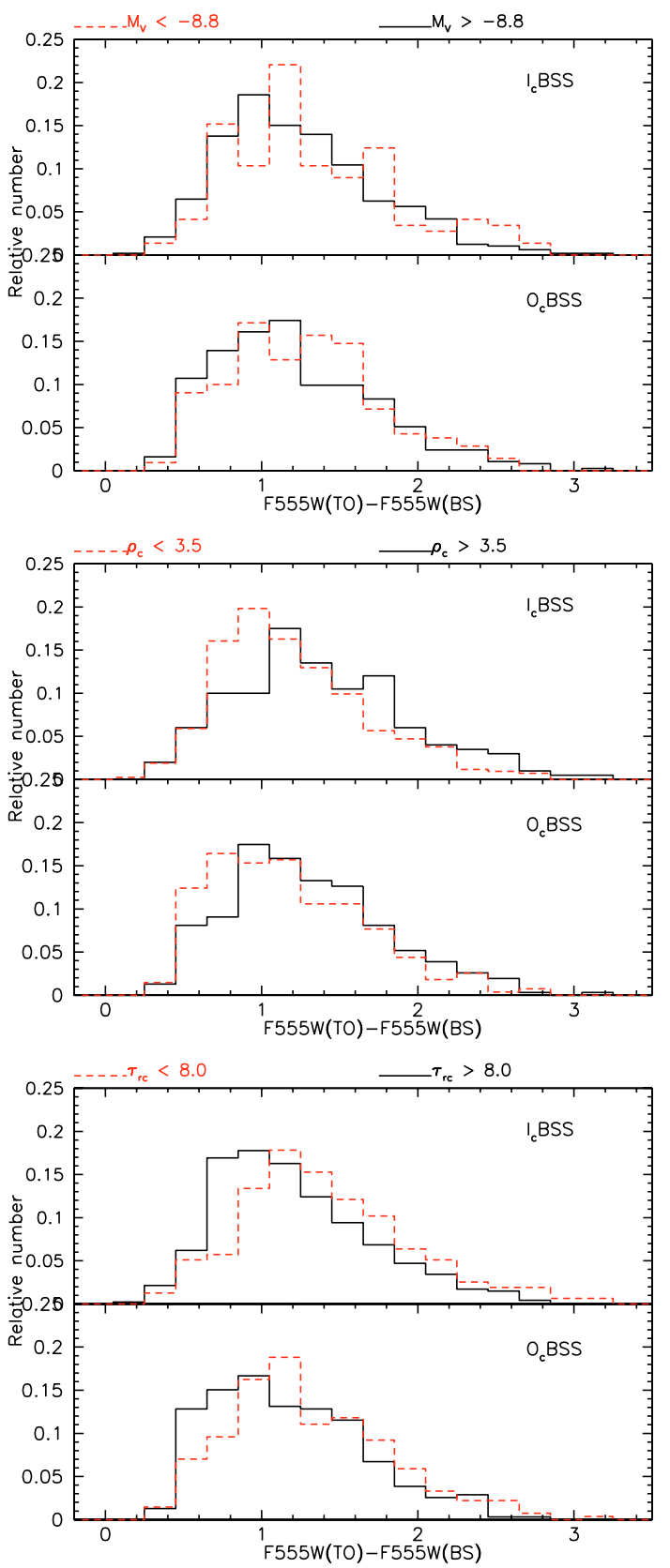

Fig. 8. Luminosity functions of $I_{\mathrm{c}} B S s$ (upper panel) and $O_{\mathrm{c}} B S S$ (lower panel) for different subsamples of globular clusters: from top to bottom clusters are divided according to $M_{V}, \rho_{\mathrm{c}}, \tau_{\mathrm{rc}}$.

differences among GCs (at least of our sample) are much smaller than the dynamical evolutionary timescale.

\subsection{Spatially-distinct populations}

In order to check whether these results depend on different BSS location with respect to the cluster centre, we repeated the experiment for the subsamples of $I_{\mathrm{c}} B S s$ and $O_{\mathrm{c}} B S s$ (in this case excluding from the analysis post core-collapse globular clusters), and $I_{\mathrm{hm}} B S s$ and $O_{\mathrm{hm}} B S s$ (for the entire sample). The total number of BSS in these regions is $1064,1369,2088$, and 335 , respectively, while the total number of BSS is 2423. It is clear that small number statistics has to be taken into account when evaluating the results coming from the $I_{\mathrm{hm}} B S s$. For these subsamples we report in Table 4 the column of the correlation matrix 

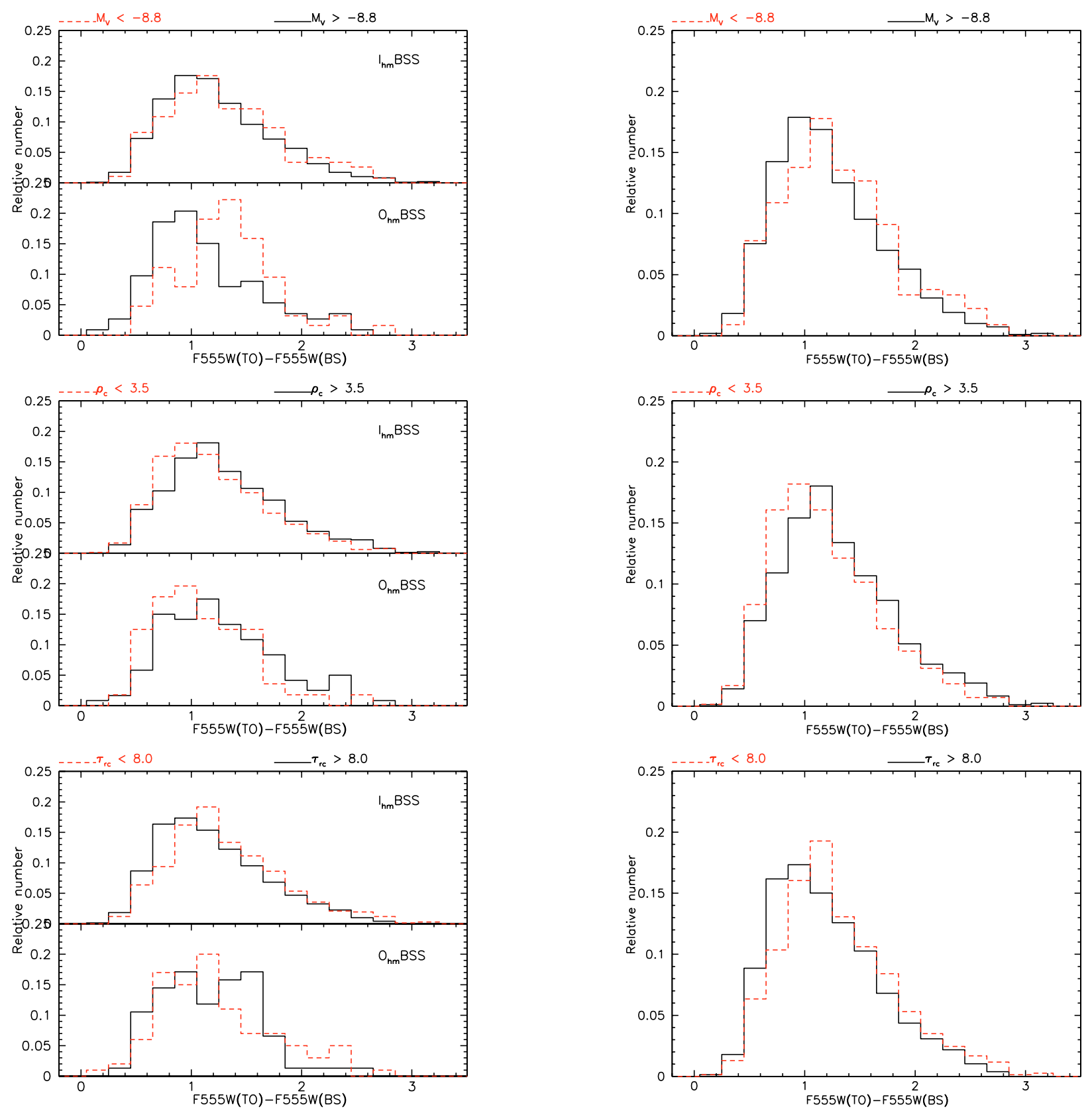

Fig. 9. Luminosity functions of $I_{\mathrm{hm}} B S s$ (upper panel) and $O_{\mathrm{hm}} B S s$ (lower panel) for different subsamples of globular clusters: from top to bottom clusters are divided according to $M_{V}, \rho_{\mathrm{c}}, \tau_{\mathrm{rc}}$.

involving the normalised number of BSS (i.e., equivalent to the second column of Table 2).

As for the complete sample, the main contributors to the total variance are the cluster luminosity, the maximum temperature along the $\mathrm{HB}$, the velocity dispersion, and the half-mass relaxation timescale, though the weight of these dependencies is slightly different for the different subsamples.

Table 4 clearly shows that $I_{\mathrm{hm}} B S s$ have properties very similar to the properties of the global sample, a result certainly not surprising, given that the field coverage is mostly marginally larger than the half-mass radius. The $I_{\mathrm{c}} B S s$ seem to be more influenced by the core and half-mass relaxation timescales (with respect to the whole BSS population) and less by the maximum temperature along the HB and the velocity dispersion. The collisional parameter also contributes less to the total variance

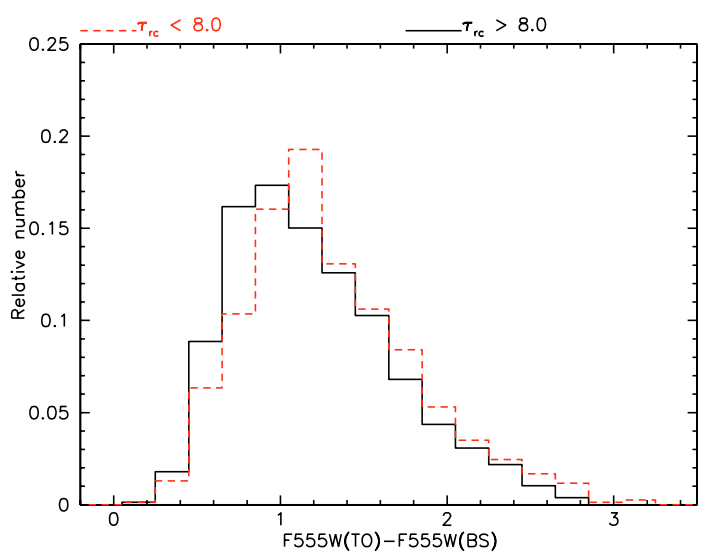

Fig. 10. Luminosity functions of different subsamples of globular clusters: from top to bottom clusters are divided according to $M_{V}, \rho_{\mathrm{c}}$, $\tau_{\mathrm{rc}}$. Here we show the whole sample of GCs, i.e. including the PCC clusters.

when looking at $I_{\mathrm{c}} B S s$ compared to the global population of BSS.

These results are also displayed in Fig. 14, where we plot the projections on the first two eigenvectors of the $I_{\mathrm{C}} B S s$ populations. We also repeated the PCA analysis excluding the velocity dispersion, since this parameter is determined for a small number of clusters. This increases the sample to $47 \mathrm{GCs}$. We find, in this case, similar dependencies.

Finally, according to the prediction of Davies et al. (2004), less luminous clusters are expected to produce more BSS via mass transfer, while in more luminous clusters, collisions are expected play a more important role. This is confirmed by our results for the BSS luminosity function, which indicate that clusters having different luminosities host different populations of 

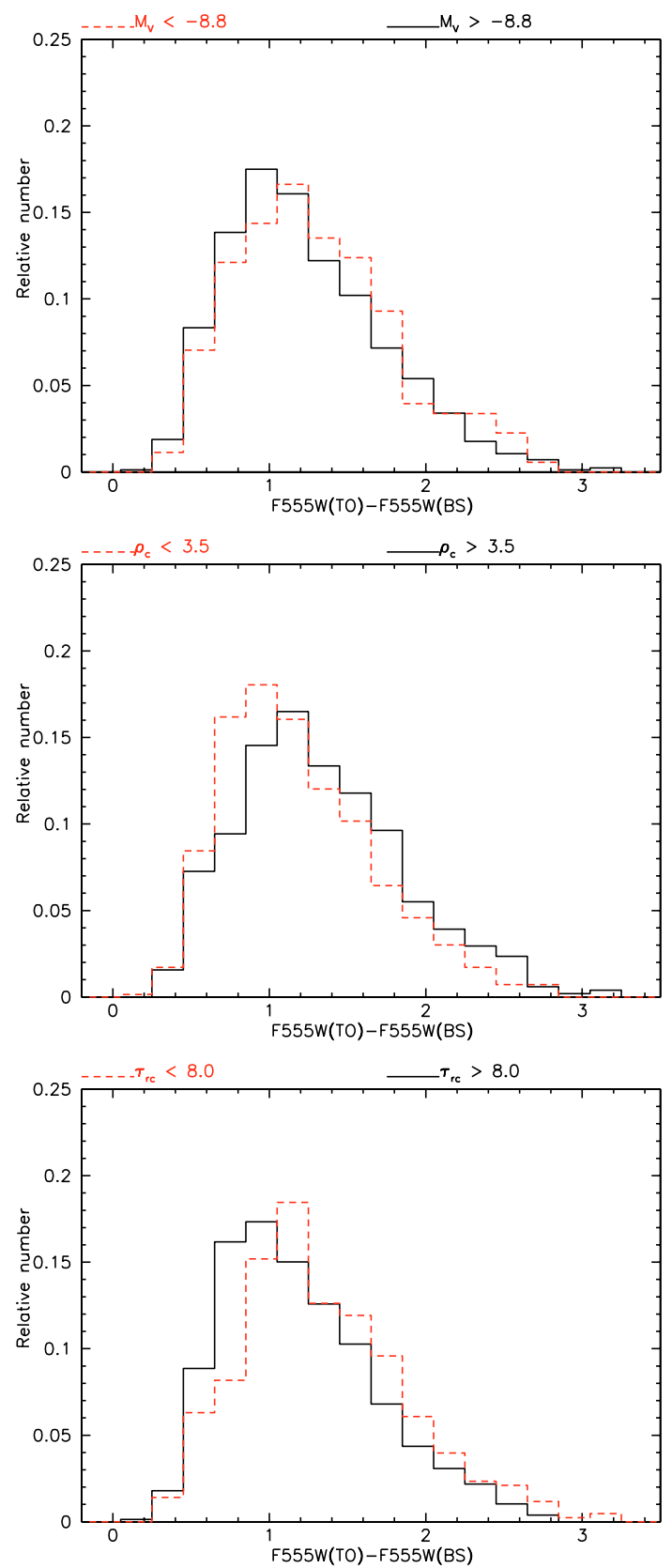

Fig. 11. Luminosity functions of different subsamples of globular clusters: from top to bottom clusters are divided according to $M_{V}, \rho_{\mathrm{c}}, \tau_{\mathrm{rc}}$. Here we show the LF of not PCC clusters.

BSS. The multivariate analysis can, thus, be used to study the dependence of the BSS inside and outside of the cluster core on the main cluster parameters for the two subsamples of bright and faint clusters. We divided our sample of non-PCC clusters according to their integrated luminosity, adopting $M_{V}=-8.8$ as a cutoff value, and extracted for each subsample the BSS located inside the core $\left(I_{\mathrm{c}} B S s\right)$ and outside the core $\left(O_{\mathrm{c}} B S s\right)$.

Table 5 contains the correlation matrix for the $I_{\mathrm{c}} B S s$ and $O_{\mathrm{c}} B S s$ in the two subsamples (labelled Bright and Faint). After excluding clusters that have one or more of the entering parameters missing we end up with 6 bright clusters and 29 faint clusters.

Interestingly enough, it is clear that in bright clusters the number of $I_{\mathrm{c}} B S S$ strongly depend on the collisional parameter and on the central density, as expected if these BSS were
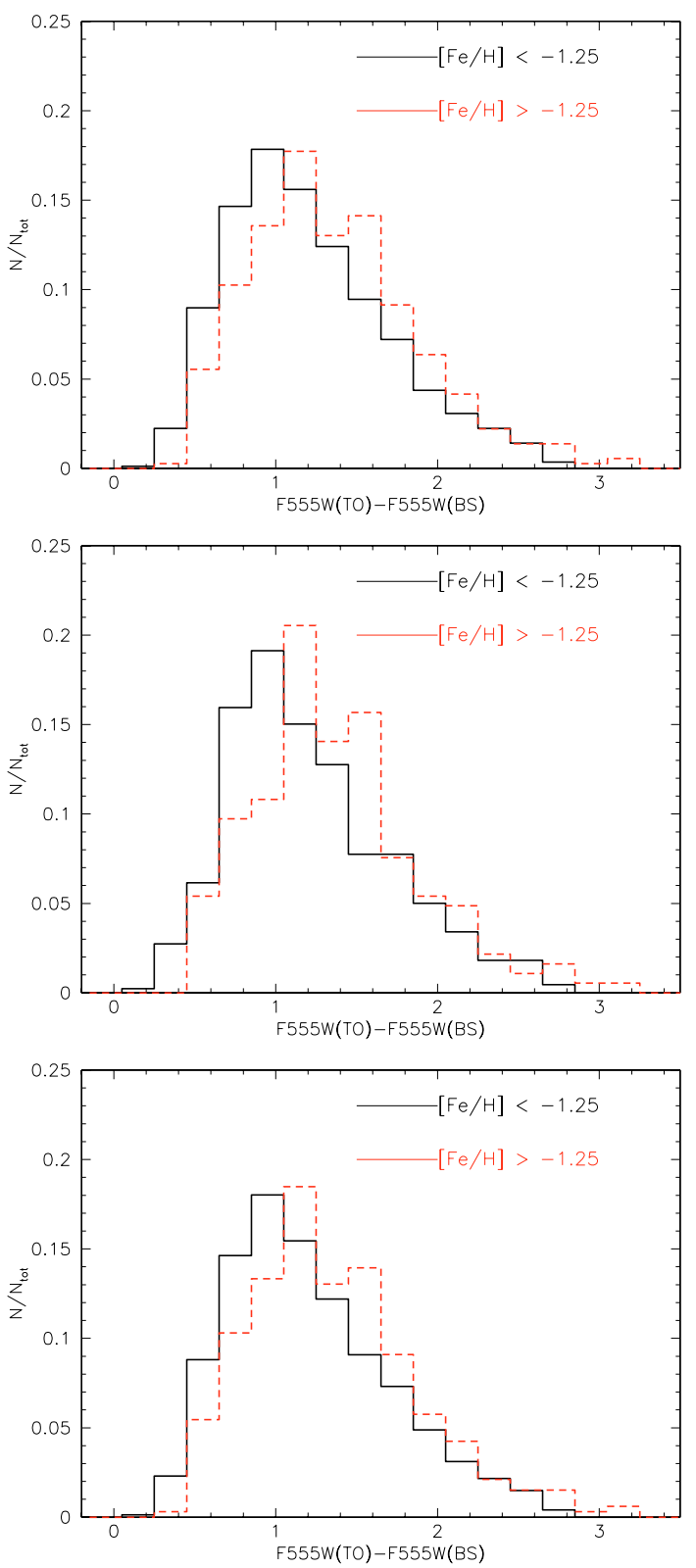

Fig. 12. Luminosity functions of different subsamples of globular clusters divided according to the metallicity: from top to bottom we show the LFs of the whole cluster sample, the LFs of the $I_{\mathrm{c}} B S s$, the LFs of $I_{\mathrm{hm}} B S s$.

produced mainly by collisions. This is in agreement with the results by Mapelli et al. (2006).

On the other hand, in faint clusters, $I_{\mathrm{c}} B S s$ are dominated by the effects of clusters luminosity and dynamical timescales, in agreement with the Davies et al. (2004) model.

\section{Discussion and conclusion}

In this paper, we analysed the properties of the BSS population in the photometrically homogeneous sample of CMDs provided by Piotto et al. (2002). The sample is homogeneous as the data come from the same observing facility (WFPC2/HST) and have been reduced applying the same procedures. However, the photometric quality in the BSS region is not the same. Because of this, we selected BSS adopting an empirical approach that separates BSS from MS stars using both an analytic expression and 

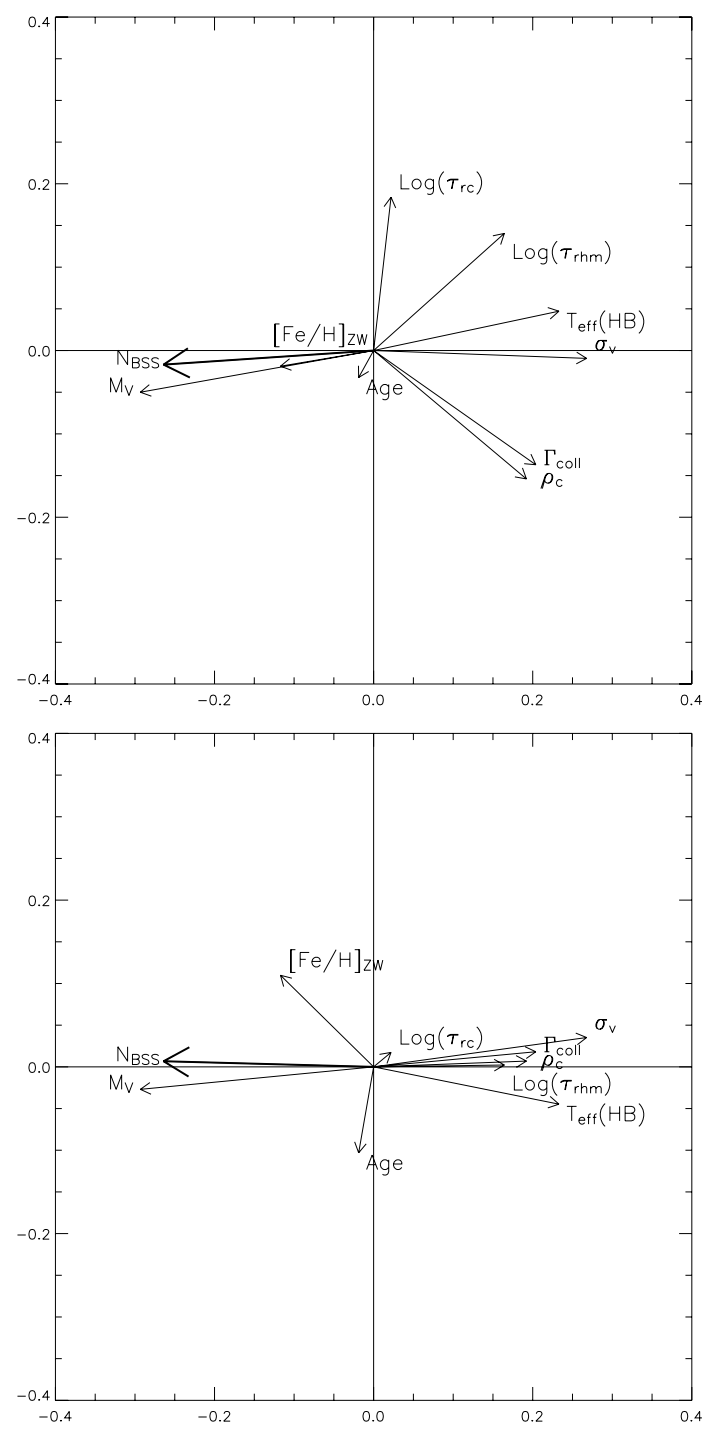

Fig. 13. Projections of the initial vectors in the first (upper) and second (lower) planes of eigenvectors. This plot refers to the total normalised population of BSS, independent of their position in the cluster.

the photometric properties of the BSS candidates. Once we selected our BSS catalogue, we divided it into subsamples of BSS inside and outside the core radius $\left(I_{\mathrm{c}} B S s\right.$ and $O_{\mathrm{c}} B S s$, respectively), and inside and outside the half-mass radius $\left(I_{\mathrm{hm}} B S s\right.$ and $O_{\mathrm{hm}} B S s$, respectively). We defined only the $I_{\mathrm{hm}} B S s$ and $O_{\mathrm{hm}} B S s$ for the whole sample of $56 \mathrm{GCs}$, while we defined the $I_{\mathrm{c}} B S s$ and $O_{\mathrm{c}} B S s$ subsamples only for $43 \mathrm{GCs}$, i.e., all the clusters but the PCC ones. We calculated the total and the relative number of BSS. We corrected the BSS counts for completeness. The relative numbers of BSS is defined as the absolute number of BSS, divided by the total luminosity of the host cluster (in units of $10^{4} L_{\odot}$ ) sampled by our WFPC2/HST images. We then investigated the possible correlations of the absolute and relative numbers of BSS with the main cluster parameters, and obtained the following results:

- Both populations of BSS inside and outside the core radius show a statistically significant anticorrelation with the cluster total luminosity. Interestingly enough, both $I_{\mathrm{c}} B S s$ and $O_{\mathrm{c}} B S s$ seem to follow the same relation. More luminous clusters have a smaller fraction of BSS. The $O_{\mathrm{c}} B S S$ show a marginal correlation with the central density: denser

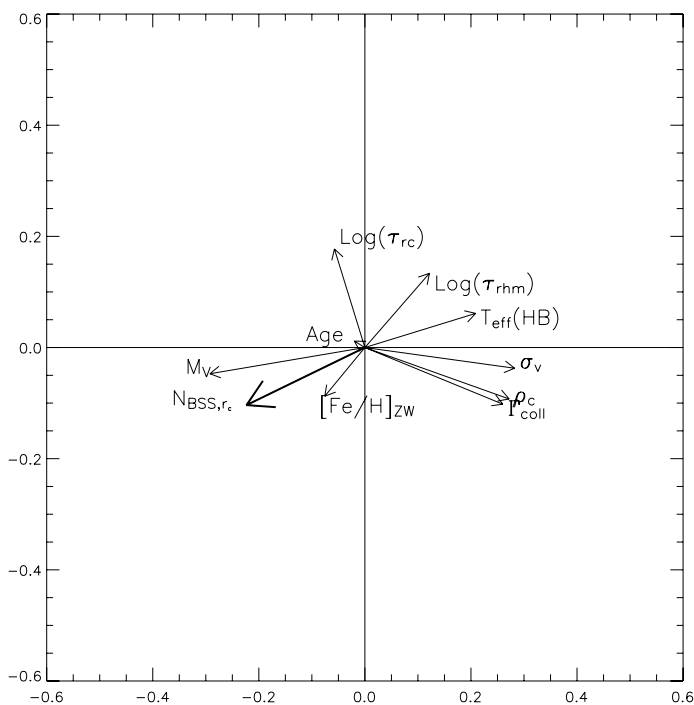

Fig. 14. Projections of the initial vectors in the first plane of eigenvectors for the population of $I_{\mathrm{c}} B S s$.

clusters tend to have a smaller fraction of BSS. This correlation could reflect the fact that in denser clusters primordial BSS evolve more rapidly and are thus presently missing, as discussed in Davies et al. (2004). There is also a marginal anticorrelation between the fraction of BSS and the half-mass relaxation time. The metallicity does not play any role, while there is a noticeable correlation between the fraction of BSS and the extension of the cluster HB: hotter HB clusters possess less BSS per unit luminosity, both inside and outside the core radius. Finally, clusters with higher central velocity dispersions host less BSS per unit luminosity.

- The samples of BSS selected on the basis of the position with respect to the cluster half-mass radius follow basically the same trends, though, in general, the dependencies on the various cluster parameters are statistically less significant.

- From the analysis of the luminosity functions of different subsamples we find that BSS have a more luminous LF peak in more massive clusters, in clusters with higher central densities and in clusters with smaller core relaxation timescales. We, therefore, confirm the results found by Piotto et al. (2004) and suspect that the different results reached by Leigh et al. (2007) are due to a different selection criterion (which does not properly account for the photometric accuracy of the data) and the lack of completeness correction. Interestingly enough, PCC cluster LFs follow the trends of normal, King-model profile clusters.

The multivariate analysis confirms the main correlations identified in the monovariate analysis. In particular, it shows that most of the variance of the sample is accounted for by the dependence of the BSS frequency on the total luminosity, the maximum temperature along the $\mathrm{HB}$, the velocity dispersion, and on the half-mass relaxation timescale. There is also a dependence on the central density and, noticeably, a marginal dependence on the collisional parameter. These dependencies are also confirmed when we perform the multivariate analysis on the BSS subsamples divided according to their location with respect to the parent cluster core radius and half-mass radius.

An interpretation of the complex observational scenario disclosed by our analysis of the dependence of BSS on the parent cluster parameters is beyond the purposes of the present paper. 
Table 2. Correlation matrix for the sample of GCs having all parameter determined (29 GCs). $N_{\mathrm{BSS}}$ is the normalised number of BSS.

\begin{tabular}{ccccccccccc}
\hline \hline & $N_{\text {BSS }}$, Norm & $M_{V}$ & $\Gamma_{\text {coll }}$ & $\tau_{\text {rc }}$ & $\tau_{\text {rhm }}$ & $\rho_{\mathrm{c}}$ & {$[\mathrm{Fe} / \mathrm{H}]$} & Age & $T_{\text {eff }}(\mathrm{HB})$ & $\sigma_{\mathrm{v}}$ \\
\hline$N_{\text {BSS }}$ & 1.000 & 0.757 & -0.457 & -0.079 & -0.494 & -0.437 & 0.322 & 0.096 & -0.620 & -0.549 \\
$M_{V}$ & 0.757 & 1.000 & -0.453 & -0.324 & -0.713 & -0.394 & 0.180 & 0.179 & -0.626 & -0.839 \\
$\Gamma_{\text {coll }}$ & -0.457 & -0.453 & 1.000 & -0.491 & -0.137 & 0.952 & -0.093 & -0.004 & 0.208 & 0.593 \\
$\tau_{\text {rc }}$ & -0.079 & -0.324 & -0.491 & 1.000 & 0.647 & -0.676 & -0.015 & -0.199 & 0.207 & 0.080 \\
$\tau_{\text {rhm }}$ & -0.494 & -0.713 & -0.137 & 0.647 & 1.000 & -0.170 & -0.208 & -0.030 & 0.349 & 0.370 \\
$\rho_{\mathrm{c}}$ & -0.437 & -0.394 & 0.952 & -0.676 & -0.170 & 1.000 & -0.120 & 0.063 & 0.193 & 0.533 \\
{$[\mathrm{Fe} / \mathrm{H}]$} & 0.322 & 0.180 & -0.093 & -0.015 & -0.208 & -0.120 & 1.000 & -0.352 & -0.567 & -0.050 \\
$\mathrm{Age}$ & 0.096 & 0.179 & -0.004 & -0.199 & -0.030 & 0.063 & -0.352 & 1.000 & 0.012 & -0.113 \\
$T_{\text {eff }}(\mathrm{HB})$ & -0.620 & -0.626 & 0.208 & 0.207 & 0.349 & 0.193 & -0.567 & 0.012 & 1.000 & 0.549 \\
$\sigma_{\mathrm{v}}$ & -0.549 & -0.839 & 0.593 & 0.080 & 0.370 & 0.533 & -0.050 & -0.113 & 0.549 & 1.000 \\
\hline & $N_{\mathrm{BSS}}$ & $M_{V}$ & $\Gamma_{\mathrm{coll}}$ & $\tau_{\mathrm{rc}}$ & $\tau_{\text {rhm }}$ & $\rho_{\mathrm{c}}$ & {$[\mathrm{Fe} / \mathrm{H}]$} & $\mathrm{Age}$ & $T_{\text {eff }}(\mathrm{HB})$ & $\sigma_{\mathrm{v}}$ \\
\hline$N_{\mathrm{BSS}}$ & 1.000 & -0.531 & 0.514 & 0.120 & 0.172 & 0.382 & 0.085 & -0.278 & 0.207 & 0.678 \\
$M_{V}$ & -0.531 & 1.000 & -0.453 & -0.324 & -0.713 & -0.394 & 0.180 & 0.179 & -0.626 & -0.839 \\
$\Gamma_{\text {coll }}$ & 0.514 & -0.453 & 1.000 & -0.491 & -0.137 & 0.952 & -0.093 & -0.004 & 0.208 & 0.593 \\
$\tau_{\text {rc }}$ & 0.120 & -0.324 & -0.491 & 1.000 & 0.647 & -0.676 & -0.015 & -0.199 & 0.207 & 0.080 \\
$\tau_{\text {rhm }}$ & 0.172 & -0.713 & -0.137 & 0.647 & 1.000 & -0.170 & -0.208 & -0.030 & 0.349 & 0.370 \\
$\rho_{\mathrm{c}}$ & 0.382 & -0.394 & 0.952 & -0.676 & -0.170 & 1.000 & -0.120 & 0.063 & 0.193 & 0.533 \\
{$[\mathrm{Fe} / \mathrm{H}]$} & 0.085 & 0.180 & -0.093 & -0.015 & -0.208 & -0.120 & 1.000 & -0.352 & -0.567 & -0.050 \\
$\mathrm{Age}$ & -0.278 & 0.179 & -0.004 & -0.199 & -0.030 & 0.063 & -0.352 & 1.000 & 0.012 & -0.113 \\
$T_{\text {eff }}(\mathrm{HB})$ & 0.207 & -0.626 & 0.208 & 0.207 & 0.349 & 0.193 & -0.567 & 0.012 & 1.000 & 0.549 \\
$\sigma_{\mathrm{v}}$ & 0.678 & -0.839 & 0.593 & 0.080 & 0.370 & 0.533 & -0.050 & -0.113 & 0.549 & 1.000 \\
\hline
\end{tabular}

Table 3. Eigenvalues and cumulative percentage contribution to the total variance of the sample GCs having all parameters determined (29 GCs).

\begin{tabular}{ccc}
\hline \hline & Eigenv. & $\%$ \\
\hline 1 & 4.0383 & 40.3833 \\
2 & 2.6056 & 66.4396 \\
3 & 1.4897 & 81.3369 \\
4 & 0.7372 & 88.7088 \\
5 & 0.4815 & 93.5236 \\
6 & 0.3204 & 96.7274 \\
7 & 0.2004 & 98.7310 \\
8 & 0.1006 & 99.7365 \\
9 & 0.0252 & 99.9887 \\
10 & 0.0011 & 100.0000 \\
\hline
\end{tabular}

Table 4. Correlation matrices for different subsamples. $N_{\text {BSS }}$ is the normalised number of BSS.

\begin{tabular}{ccccc}
\hline \hline & $r<r_{\mathrm{c}}$ & $r>r_{\mathrm{c}}$ & $r<r_{\mathrm{hm}}$ & $r>r_{\mathrm{hm}}$ \\
& $N_{\mathrm{BSS}}$ & $N_{\mathrm{BSS}}$ & $N_{\mathrm{BSS}}$ & $N_{\mathrm{BSS}}$ \\
\hline$N_{\mathrm{BSS}}$ & 1.000 & 1.000 & 1.000 & 1.000 \\
$M_{V}$ & 0.875 & 0.703 & 0.794 & 0.479 \\
$\Gamma_{\text {coll }}$ & -0.451 & -0.357 & -0.434 & -0.141 \\
$\tau_{\mathrm{rc}}$ & -0.350 & -0.272 & -0.098 & -0.091 \\
$\tau_{\text {rhm }}$ & -0.637 & -0.621 & -0.541 & -0.493 \\
$\rho_{\mathrm{c}}$ & -0.476 & -0.381 & -0.426 & -0.181 \\
{$[\mathrm{Fe} / \mathrm{H}]$} & 0.333 & 0.541 & 0.298 & 0.446 \\
$\mathrm{Age}$ & 0.093 & 0.097 & 0.113 & 0.000 \\
$T_{\text {eff }}(\mathrm{HB})$ & -0.616 & -0.526 & -0.624 & -0.371 \\
$\sigma_{\mathrm{v}}$ & -0.640 & -0.451 & -0.570 & -0.238 \\
\hline
\end{tabular}

However, we hope that the various relations above discussed can help interpreting the origin of BSS in globular clusters.

Acknowledgements. We thank Dr. Luca Sbordone for providing the centres of the clusters. A.M. acknowledges the kind hospitality of the Institute of Astronomy (Cambridge) where this work started, as well as the European EARA network for travel funding.
Table 5. Correlation matrices for the two subsamples of bright and faint GCs. $N_{\text {BSS }}$ is the normalised number of BSS.

\begin{tabular}{ccccc}
\hline \hline & Bright & Faint & Bright & Faint \\
& $N_{I_{\mathrm{c}} B S s}$ & $N_{I_{\mathrm{c}} B S s}$ & $N_{O_{\mathrm{c}} B S s}$ & $N_{O_{\mathrm{c}} B S s}$ \\
\hline$N_{\mathrm{BSS}}$ & 1.000 & 1.000 & 1.000 & 1.000 \\
$M_{V}$ & 0.283 & 0.561 & -0.363 & 0.565 \\
$\Gamma_{\text {coll }}$ & 0.685 & 0.182 & 0.348 & -0.329 \\
$r_{\mathrm{c}}$ & -0.518 & -0.156 & -0.576 & 0.278 \\
$r_{\mathrm{hm}}$ & 0.135 & -0.004 & -0.234 & 0.422 \\
$\tau_{\mathrm{rc}}$ & -0.876 & -0.630 & -0.657 & -0.171 \\
$\tau_{\mathrm{rhm}}$ & 0.332 & -0.486 & -0.283 & -0.239 \\
$\rho_{\mathrm{c}}$ & 0.725 & 0.212 & 0.415 & -0.302 \\
{$[\mathrm{Fe} / \mathrm{H}]$} & 0.236 & 0.317 & -0.057 & 0.220 \\
$\mathrm{Age}$ & 0.027 & -0.115 & 0.231 & 0.054 \\
$T_{\mathrm{eff}}(\mathrm{HB})$ & -0.512 & -0.334 & -0.020 & -0.340 \\
\hline
\end{tabular}

\section{Appendix A: BSS selection}

A photometric database of 74 Galactic GCs has been published by Piotto et al. (2002). All the clusters were observed in the $F 439 \mathrm{~W}$ and $F 555 \mathrm{~W}$ photometric bands pointing the HST/WFPC2 camera to the central core. A detailed discussion of the reduction steps, and of the procedures to transform instrumental magnitudes into both the HST F439W and F555W flight system, and the standard Johnson-Cousin $B$ and $V$ system are presented in Piotto et al. (2002). For all the 74 GCs, artificial star experiments have been run to estimate the completeness of the star counts and the photometric internal errors in all the relevant branches of the CMD, including the BSS region. Details on the artificial star test are in Piotto et al. (2002). The entire photometric catalogue, as well as the completeness measurements as a function of magnitude, can be found at the Padova globular cluster group web page (http://dipastro. astro.unipd.it/globulars).

The BSS were extracted directly through visual inspection of the CMD of each cluster. BSS are clearly present in all the 74 GCs of our database. However, 18 of the GCs were too low on the galactic plane or too close to the Galactic bulge, and, 
Table A.1. Example of a BSS candidate table for the cluster NGC 4833: Col. 1 gives a star identification number; Cols. 2 and 3 give the position on the chip; Cols. 4 and 5, the $V$ and $B$ standard magnitudes (reddening corrected); Cols. 6 and 7, the $F 555 W$ and $F 439 W$ magnitudes in the HST flight system (reddening corrected); Cols. 8 and 9, the photometric errors given by ALLFRAME; Cols. 10 and 11 the, $V$ and $B$ standard magnitudes (before the reddening correction); Cols. 12 and 13 , the $F 555 W$ and $F 439 W$ flight magnitudes (before the reddening correction); Cols. 14 and 15 , the $\chi$ and sharp parameters as given by ALLFRAME; and Col. 16 the chip number ( 1 for PC, and 2, 3, 4 for WF2, WF3, WF4, respectively).

\begin{tabular}{|c|c|c|c|c|c|c|c|}
\hline$\overline{\mathrm{ID}}$ & $x$ & $y$ & $\bar{V}$ & $\bar{B}$ & $\overline{F 555 W}$ & 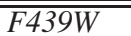 & $\overline{\sigma_{F 555 W}}$ \\
\hline 20 & 397.925 & 55.752 & 16.15 & 16.30 & 16.16 & 16.30 & 0.03 \\
\hline 510 & 257.404 & 232.309 & 16.21 & 16.22 & 16.21 & 16.22 & 0.03 \\
\hline 1452 & 535.892 & 454.795 & 16.25 & 16.47 & 16.26 & 16.47 & 0.03 \\
\hline 220 & 59.999 & 114.546 & 16.33 & 16.48 & 16.34 & 16.48 & 0.04 \\
\hline 974 & 193.712 & 420.964 & 16.37 & 16.41 & 16.37 & 16.41 & 0.05 \\
\hline 1930 & 346.796 & 686.376 & 16.38 & 16.55 & 16.39 & 16.56 & 0.04 \\
\hline 903 & 185.030 & 384.696 & 16.52 & 16.73 & 16.53 & 16.73 & 0.04 \\
\hline & $V_{\mathrm{nr}}$ & $B_{\mathrm{nr}}$ & $F 555 W_{\mathrm{nr}}$ & $\overline{F 439 W_{\mathrm{n}}}$ & $\chi$ & sharp & chip \\
\hline & 17.19 & 17.66 & 17.21 & 17.68 & 3.595 & 0.061 & 4 \\
\hline & 17.25 & 17.60 & 17.27 & 17.61 & 3.944 & 0.003 & 3 \\
\hline & 17.29 & 17.82 & 17.31 & 17.85 & 3.692 & 0.010 & 2 \\
\hline & 17.37 & 17.84 & 17.39 & 17.86 & 3.866 & 0.009 & 4 \\
\hline & 17.41 & 17.78 & 17.43 & 17.79 & 5.721 & 0.039 & 3 \\
\hline & 17.42 & 17.91 & 17.44 & 17.93 & 3.111 & -0.005 & 2 \\
\hline & 17.56 & 18.08 & 17.58 & 18.11 & 3.862 & 0.023 & 3 \\
\hline
\end{tabular}

therefore, badly contaminated by background and/or foreground stars, or affected by differential reddening, to allow a reliable selection of BSS. For these clusters, follow-up, second epoch images to measure proper motions would be of fundamental importance to extract the GC members (as done in Bedin et al. 2001), including BSS. For 3 clusters (namely NGC 5927, NGC 6287, and NGC 6864), the BSS were selected from the CMD of the PC camera only, to minimise the contamination by field stars.

Defining a BSS sample from the optical CMD is arbitrary, mainly due to the difficulty in separating faint BSS from normal main sequence (MS) stars at the turnoff (TO) level. At the high density of the GC central regions, the probability of having a casual superposition of the image of two or more stars (blending) is high (in this respect, if we are interested in measuring the relative number of BSS with respect to some other reference stellar population of the parent cluster, the artificial star experiments become an essential complement of the catalogue).

In our selection procedure, we tried to extract the BSS from a region in the CMD well separated from the MS. In fact, close to the MS, some contamination by casual blends is unavoidable. Moreover, as already mentioned in Bailyn \& Pinsonneault (1995), a possible selection effect could come from the fact that fainter stars are generally required to be bluer than the MS to qualify as BSS, whereas theoretical expectations would suggest fainter BSS to be redder than their brighter counterparts. For this reason, particularly when we are interested in comparing BSS luminosity functions among different clusters, or with their theoretical counterpart, it is important to work only in the luminosity range in which the BSS sequence is clearly separated from the main sequence. One common practice is to use only BSS brighter than the TO by some predefined quantity. In this respect, an internally consistent measurement of the TO colours and magnitudes of most of the clusters used in the present paper can be found in De Angeli et al. (2005).

We decided to adopt a slightly different criterion: we did not simply apply a cut in magnitude taking the TO as reference point, as this would have excluded from our catalogue many BSS that are definitely bluer, but not much brighter than the TO, and would instead have left us with many possible blendings slightly brighter than the subgiant branch (SGB). We instead defined two

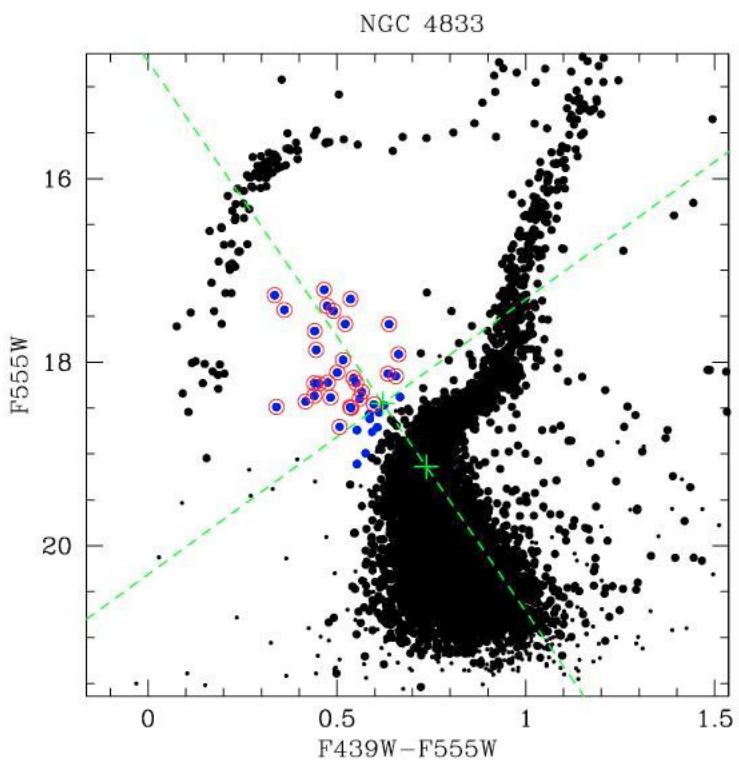

Fig. A.1. The $F 555 W$ vs. $F 439 W-F 555 W$ (HST flight system) colour-magnitude diagrams of cluster NGC 4833. Example of our twostep selection process.

reference lines on each CMD: one approximately parallel to the ZAMS (assuming a slope equal to 6.0) passing through the TO, and another one approximately parallel to the SGB (assuming a slope equal to -3.0 ) crossing the first line $0.7 \mathrm{mag}$ above the TO. We proceeded by first selecting the BSS candidates by visual inspection of the CMD and then refining our catalogue by accepting only stars bluer than the second line defined above (the one parallel to the SGB). The process is graphically shown in Fig. A.1 for the cluster NGC 4833. The cluster CMD is shown with black dots, blue full circles are the BSS candidates extracted by visual inspection, those circled in red are the confirmed ones lying on the blue side of the green line approximately parallel to the SGB. The turnoff and the point along the ZAMS 0.7 mag brighter are marked by green crosses.

Unfortunately, six clusters among the 56 selected for this study, did not have an estimate of the turnoff magnitude and 
colour. For these, therefore, we publish the list of candidates extracted by visual inspection of the CMD only. The clusters are NGC 6229, NGC 6293, NGC 6304, NGC 6441, NGC 6569, and NGC 6642.

We believe that the distinction between bright BSS and horizontal branch stars is quite straightforward in the vast majority of the cases and, therefore, we do not consider it necessary to define a boxed area in the CMD where the BSS should be extracted from.

The whole catalogue will be available in electronic form from Centre de Données Astronomiques de Strasbourg (CDS, http://cdsweb.u-strasbg.fr). The BSS candidates are listed in one table per cluster. A detailed description of the photometric measurements and calibration procedures is in Piotto et al. (2002); the tables with position, magnitudes, fitting parameters, and errors have the same format of the tables published by Piotto et al. (2002) for HST snapshot catalogue. The content of the electronic tables is also described in detail in the file ReadMe available on-line. The first few lines of the table for NGC 4833 are given as an example in Table A.1 (the header will not be included in the electronic tables).

\section{References}

Ahumada, J., \& Lapasset, E. 1995, A\&AS, 109, 375

Ahumada, J. A., \& Lapasset, E. 2007, A\&A, 463, 789

Bailyn, C. D. 1995, ARA\&A, 33, 133

Bailyn, C. D., \& Pinsonneault, M. H. 1995, ApJ, 439, 705

Beccari, G., Ferraro, F. R., Possenti, A., et al. 2006, AJ, 131, 2551

Bedin, L. R., Anderson, J., King, I. R., \& Piotto, G. 2001, ApJ, 560, L75

Bellazzini, M., Ferraro, F. R., \& Fusi Pecci, F. 1992, Mem. Soc. Astron. Ital., 63, 111

Brosche, P., \& Lentes, F.-T. 1984, A\&A, 139, 474

Carney, B. W., Latham, D. W., Laird, J. B., Grant, C. E., \& Morse, J. A. 2001, AJ, 122,3419

Carretta, E., \& Gratton, R. G. 1997, A\&AS, 121, 95

Clark, L. L., Sandquist, E. L., \& Bolte, M. 2004, AJ, 128, 3019

Davies, M. B., Piotto, G., \& De Angeli, F. 2004, MNRAS, 349, 129
De Angeli, F., Piotto, G., Cassisi, S., et al. 2005, AJ, 130, 116

de Marchi, F., De Angeli, F., Piotto, G., Carraro, G., \& Davies, M. B. 2006, A\&A, 459, 489

Djorgovski, S. 1988, in The Harlow-Shapley Symposium on Globular Cluster Systems in Galaxies, ed. J. E. Grindlay, \& A. G. D. Philip, IAU Symp., 126, 333

Djorgovski, S. 1991, in The Formation and Evolution of Star Clusters, ed. K. Janes, ASP Conf. Ser., 13, 112

Djorgovski, S. 1993, in Structure and Dynamics of Globular Clusters, ed. S. G. Djorgovski, \& G. Meylan, ASP Conf. Ser., 50, 373

Djorgovski, S., \& Meylan, G. 1994, AJ, 108, 1292

Djorgovski, S., Piotto, G., \& Capaccioli, M. 1993, AJ, 105, 2148

Ferraro, F. R., D’Amico, N., Possenti, A., Mignani, R. P., \& Paltrinieri, B. 2001, ApJ, 561, 337

Ferraro, F. R., Paltrinieri, B., Fusi Pecci, F., et al. 1997, A\&A, 324, 915

Ferraro, F. R., Sollima, A., Rood, R. T., et al. 2006, ApJ, 638, 433

Harris, W. E. 1996, AJ, 112, 1487

King, I. R. 2002, Introduction to Classical Stellar Dynamics (Moscow URSS)

Leigh, N., Sills, A., \& Knigge, C. 2007, ApJ, 661, 210

Mapelli, M., Sigurdsson, S., Colpi, M., et al. 2004, ApJ, 605, L29

Mapelli, M., Sigurdsson, S., Ferraro, F. R., et al. 2006, MNRAS, 373, 361

McCrea, W. H. 1964, MNRAS, 128, 147

Momany, Y., Held, E. V., Saviane, I., et al. 2007, A\&A, 468, 973

Piotto, G., King, I. R., Djorgovski, S. G., et al. 2002, A\&A, 391, 945

Piotto, G., De Angeli, F., King, I. R., et al. 2004, ApJ, 604, L109

Pryor, C., \& Meylan, G. 1993, in Structure and Dynamics of Globular Clusters, ed. S. G. Djorgovski, \& G. Meylan, ASP Conf. Ser., 50, 357

Recio-Blanco, A., Aparicio, A., Piotto, G., De Angeli, F., \& Djorgovski, S. G. 2006, A\&A, 452, 875

Recio-Blanco, A., Piotto, G., De Angeli, F., et al. 2005, A\&A, 432, 851

Rosenberg, A., Aparicio, A., Saviane, I., \& Piotto, G. 2000a, A\&AS, 145, 451

Rosenberg, A., Piotto, G., Saviane, I., \& Aparicio, A. 2000b, A\&AS, 144, 5

Sabbi, E., Ferraro, F. R., Sills, A., \& Rood, R. T. 2004, ApJ, 617, 1296

Sandage, A. R. 1953, AJ, 58, 61

Sbordone, L. 2001, Master's thesis, Università degli Studi di Padova

Trager, S. C., Djorgovski, S., \& King, I. R. 1993, in Structure and Dynamics of Globular Clusters, ed. S. G. Djorgovski, \& G. Meylan, ASP Conf. Ser., 50, 347

Trager, S. C., King, I. R., \& Djorgovski, S. 1995, AJ, 109, 218

Warren, S. R., Sandquist, E. L., \& Bolte, M. 2006, ApJ, 648, 1026

Zaggia, S. R., Piotto, G., \& Capaccioli, M. 1997, A\&A, 327, 1004

Zinn, R., \& West, M. J. 1984, ApJS, 55, 45 